Please do not remove this page

RMIT

UNIVERSITY

\title{
Minimising total flow-time on two parallel machines with planned downtimes and resumable jobs
}

Ozlen, Melih; Webster, Scott

https://researchrepository.rmit.edu.au/esploro/outputs/9921857970901341/filesAndLinks?institution=61RMIT_INST\&index=null

Ozlen, M., \& Webster, S. (2010). Minimising total flow-time on two parallel machines with planned downtimes and resumable jobs. International Journal of Production Research, 48(1), 201-226.

https://doi.org/10.1080/00207540802446795

Document Version: Accepted Manuscript

Published Version: https://doi.org/10.1080/00207540802446795

Repository homepage: https://researchrepository.rmit.edu.au

(c) 2010 Taylor \& Francis

Downloaded On 2023/04/26 22:05:55 +1000

Please do not remove this page 
Thank you for downloading this document from the RMIT Research Repository.

The RMIT Research Repository is an open access database showcasing the research outputs of RMIT University researchers.

RMIT Research Repository: http://researchbank.rmit.edu.au/

\section{Citation:}

Ozlen, M and Webster, S 2010, 'Minimising total flow-time on two parallel machines with planned downtimes and resumable jobs', International Journal of Production Research, vol. 48, no. 1, pp. 201-226.

See this record in the RMIT Research Repository at:

https://researchbank.rmit.edu.au/view/rmit:6418

Version: Accepted Manuscript

Copyright Statement:

(C) 2010 Taylor \& Francis

\section{Link to Published Version:}

http://dx.doi.org/10.1080/00207540802446795 


\title{
Minimizing Total Flowtime on Two Parallel Machines with Planned Downtimes and Resumable Jobs
}

\author{
Melih Özlen* \\ Department of Industrial Engineering, \\ Hacettepe University, 06800, Ankara, Turkey, \\ Phone: +90 3122978950 \\ Fax: +90 3122978953 \\ E-mail: mozlen@hacettepe.edu.tr \\ Scott Webster \\ Whitman School of Management, \\ 721 University Ave., Syracuse, NY 13244-2450, \\ Phone: +13154433751 \\ Fax: +1 3154421461 \\ E-mail: stwebste@syr.edu
}

*Corresponding Author 


\title{
Minimizing Total Flowtime on Two Parallel Machines with Planned Downtimes and Resumable Jobs
}

\begin{abstract}
This study considers the flowtime minimization problem on two identical parallel machines, where the machines are subject to planned downtimes. Jobs that are interrupted due to machine downtimes may resume processing once the machine becomes available without incurring additional costs or setup times. We identify optimality properties, develop upper and lower bounding procedures, and present a branch and bound methodology based on the proposed properties and bounds. The computational efficiency is tested on problem instances with up to 35 jobs.
\end{abstract}

Keywords: Scheduling, Identical Parallel Machines, Planned Downtimes, Flowtime, Preemption. 


\section{Introduction}

Most of the scheduling literature considers problems in environments where machines are assumed to be continuously available, i.e., jobs can be scheduled anytime. However, in order to work without unexpected breakdowns, machines may require certain downtimes as maintenance or cooling periods. Previously scheduled jobs, known labor or material shortages can also cause the machines to be unavailable within known time windows. Generally, all the above mentioned reasons cause the machines to be scheduled with planned downtimes.

Figure 1.1 illustrates two example schedules with planned downtimes. The first example in Figure 1.1 comes from a machine tool manufacturer in northern Illinois. This firm operates the machine shop between the hours of 4:00 a.m. and 4:00 p.m. seven days per week. Machine maintenance is normally scheduled during the off-hours (i.e., between 4:00 p.m. and 4:00 a.m.). The second example in Figure 1.1 is based on a machine shop that operates 24 hours per day, seven days per week. In this example, maintenance activities are not synchronous, but are timed according to the planned needs of the machines and the availability of maintenance crews. In both examples, a job that begins, but does not complete, processing prior to a downtime is scheduled to resume after the downtime.

\section{INSERT FIGURE 1.1 HERE}

Scheduling problems with planned downtimes have become a focus area of research. Lee, Lei, and Pinedo (1997), as part of their survey in trends on scheduling, briefly cover research on scheduling problems with planned downtimes. Sanlaville and Schmidt (1998), and Schmidt (2000) provide focused reviews of research on scheduling with planned downtimes. The authors summarize available solution methodologies for alternative scheduling models, and report known complexity results. 
This study concentrates on the problem of minimizing flowtime on two identical parallel machines with planned downtimes, though we will introduce the problem in its more general form where the number of machines is arbitrary. Minimizing flowtime, which is equivalent to minimizing the average number of jobs in the system, is an efficiency oriented criterion that is widely considered in the literature. The following review of the literature is limited to research on the flowtime criterion.

Alternative regimes for job preemption have been studied in the literature:

1. Preempt-resume: a job can be preempted without loss of work time (i.e., resumable jobs)

2. Preempt-resume with fixed setup: a preempted job requires a fixed setup time to resume processing.

3. Preempt-repeat: a preempted job must start over from the beginning.

The single machine preempt-repeat problem with a single planned downtime was first studied by Adiri et al. (1989). They show that the problem is NP-Hard. Lee and Liman (1992) study the same problem. They show that the flowtime of a schedule constructed with the shortest-processing-time (SPT) rule will be no more than 2/7 larger than the optimum. Sadfi (2005) improves this worst case performance bound to $3 / 17$ by proposing a 2-OPT exchange heuristic. Sadfi et al. (2002) propose a pseudo-polynomial time dynamic programming algorithm that returns an optimal schedule for the problem.

Graves and Lee (1999) study a problem where a single downtime, as well as a number of jobs, must be scheduled. The downtime must be complete by a pre-specified deadline, and preempted jobs can resume processing after a fixed setup time (i.e., preempt-resume with fixed setup). They show that problem complexity depends on the value of the downtime deadline. When the deadline is large, the problem is NP-hard, and when the deadline is small, the problem 
can be optimally solved with the SPT rule. Others researchers who have studied problems that include scheduling downtimes include Qi et al. (1999), Lee and Chen (2000), Chen (2006), Lee and Leon (2001), and He et al. (2005). Qi et al. (1999) and Chen (2006) study job and downtime scheduling on a single machine, and Lee and Chen (2000) consider the problem of scheduling jobs and downtimes on parallel machines. Lee and Leon (2001) and He et al. (2005) study a job and downtime single machine scheduling problem where the machine rate changes after the scheduled downtime.

The case of multiple planned downtimes on a single machine was considered first by Lee (1996). He shows that the preempt-resume problem can be optimally solved with the preemptive SPT (PSPT) rule. The PSPT rule schedules jobs in SPT order, but jobs are preempted and resumed around planned downtimes. If the objective is to minimize total weighted flowtime, then the problem is NP-hard in the ordinary sense (Lee (1996)). Wang et al. (2005) propose heuristics for this problem, and analyze their worst-case performance. Kacem and Chu ((2008a), (2008b)) propose a branch and bound procedure and develop worst-case analysis of heuristics for the non-preemptive problem of minimization of total weighted flowtime on a single machine with a single downtime. Kacem et al. (2008) describe and evaluate several optimization procedures for the same problem.

Chen (2006) considers the single machine preempt-repeat problem with multiple planned downtimes. The duration of planned downtimes and the time between planned downtimes is assumed to be fixed (e.g., periodic maintenance). This problem is NP-hard (Adiri et al. (1989)). The author proposes a branch and bound algorithm ${ }^{1}$ and develops a heuristic. Kacem et al. (2005) consider the problem of Chen (2006), but allow for arbitrary planned downtimes (e.g.,

\footnotetext{
${ }^{1}$ Batun and Azizoglu (2008) identify an error in this paper, of which the main consequence is that the branch and bound algorithm will not necessarily return an optimal solution.
} 
downtimes are not necessarily periodic). They propose a branch and bound algorithm and a dynamic programming algorithm for the problem. Batun and Azizoglu (2008) propose an alternative branch and bound algorithm for the problem.

There is little published work on the parallel machine total flowtime problem with planned downtimes. Lee and Liman (1993) consider the two parallel machine flowtime problem. One machine is continuously available and the other machine is only available for a finite time interval. Preemption is not allowed. The authors prove that the problem is NP-hard and propose a pseudo-polynomial time dynamic programming algorithm. Mosheiov (1994) studies the identical parallel machine problem where each machine has a single time interval during which it is available. This problem is NP-hard (Lee and Liman (1993)). He analyzes two heuristics for the problem and shows that the heuristics are asymptotically optimal as the number of jobs approaches infinity (i.e., the flowtime of the heuristic solution divided by the optimum approaches 1 as problem size increases). Lee (1996) studies a two parallel machine problem where there is a single planned downtime on one machine and the other machine is continuously available. He shows that the weighted flowtime problem is NP-hard when either preempt-resume or preempt-repeat is assumed.

In this paper, our aim is to minimize flowtime on identical parallel machines with multiple planned downtimes and resumable jobs. Our interest in this problem is motivated by the question of how to design robust production / delivery systems, and for a given design, how to plan the transformation and flow of materials to achieve greater resiliency. Issues associated with managing and planning for supply chain disruptions (e.g., downtimes) are garnering an increasing share of top management attention, no doubt spurred by recent catastrophic events. 
We focus on a model with resumable jobs, which tends to apply, for example, when a job is comprised of multiple copies of a discrete part (e.g., first shift continues producing parts for a job that was partially completed during the second shift the prior eve). The existence of this type of structure in practice is one reason why we examine a model with the preempt-resume feature. Another reason is based on our expectation that the preempt-resume model, while NP-hard (as shown in Section 2), is likely to be more tractable than the other two regimes noted above. Tractability is often a useful feature in the study of an issue-it increases the likelihood of exposing theoretical properties that form the basis of insight into more complicated models.

The rest of the paper is organized as follows. In Section 2, we formally define the problem and provide complexity results. In Section 3, we present properties of optimal solutions, upper and lower bounds, and our branch and bound procedure. Section 4 covers the results of our computational experiments, and we offer conclusions in Section 5. All proofs are located in the appendix.

\section{Problem and Complexity}

We assume job processing times, denoted $p_{j}$ for job $j$, are deterministic. Job setup times are sequence-independent and thus included in the job processing times. There are $n$ jobs available at time zero to be scheduled on $m$ identical parallel machines, and jobs are indexed in order of smallest-to-largest processing time. We note that while most of the properties that we present apply to problems with an arbitrary number of identical parallel machines, one of the properties that we deploy in our solution algorithm (i.e., Property 3.4) applies to the two-machine problem. For this reason, our discussion of algorithms will largely center on the case of two machines. 
The schedule of the planned downtimes is known in advance. $T_{i k}$ is the start time and $D_{i k}$ is the duration of the $k^{\text {th }}$ planned downtime on machine $i$, and $n_{i}$ is the number of planned downtimes on machine $i$. Figure 1.1 portrays Gantt charts that illustrate two cases of planned downtimes.

1. Synchronous-periodic: the schedule of uptimes and downtimes is the same on each machine, and follows a repetitive pattern

2. Asynchronous: machines have independent downtime schedules

We use the notation introduced by Lee (1996) for specifying problems with availability constraints within the standard three-field representation scheme, $\alpha|\beta| \gamma$, for scheduling problems. In this scheme, $\alpha$ specifies the machine environment, $\beta$ represents the problem characteristics, and $\gamma$ denotes the criteria to be optimized. For example, $\mathrm{r}-\mathrm{a}$ in the $\beta$ field indicates the presence of resumable availability constraints (Lee 1996). This means that if an in-process job cannot finish before a planned downtime on the machine, then it can continue after the machine is available again. In addition, an s-p in the $\beta$ field indicates that downtimes are synchronized across machines and are periodic. Problems considered in this paper are listed below.

- $\mathrm{P}|\mathrm{r}-\mathrm{a}, \mathrm{s}-\mathrm{p}| \Sigma C_{j}$ : identical parallel machines, total flowtime problem with resumable jobs and multiple fixed synchronous and periodic maintenance tasks; the problem with $m=2$ is $\mathrm{P} 2|\mathrm{r}-\mathrm{a}, \mathrm{s}-\mathrm{p}| \Sigma C_{j}$

- $\mathrm{P}|\mathrm{r}-\mathrm{a}| \Sigma C_{j}$ : identical parallel machines, total flowtime problem with resumable jobs and multiple fixed maintenance tasks; the problem with $m=2$ is $\mathrm{P} 2|\mathrm{r}-\mathrm{a}| \Sigma C_{j}$

Note that problem $\mathrm{P}|\mathrm{r}-\mathrm{a}| \Sigma C_{j}$ includes problem $\mathrm{P}|\mathrm{r}-\mathrm{a}, \mathrm{s}-\mathrm{p}| \Sigma C_{j}$ as a special case. 
Lee (1996) shows that $1|\mathrm{r}-\mathrm{a}| \sum w_{j} C_{j}$ is NP-hard, and thus $\mathrm{P} 2|\mathrm{r}-\mathrm{a}| \sum w_{j} C_{j}$ is NP-hard. He describes a pseudo-polynomial time DP algorithm for $\mathrm{P} 2|\mathrm{r}-\mathrm{a}| \sum w_{j} C_{j}$, which implies that the problem is NP-hard in the ordinary sense. As noted above, Lee (1996) also shows that $1|\mathrm{r}-\mathrm{a}| \Sigma C_{j}$ can be solved in polynomial time by applying the PSPT rule. Lee and Yu (2008) study the two identical parallel machines total flowtime problem with resumable jobs and a single downtime during which all machines are simultaneously down. They prove that this problem is NP-hard, which implies that the more general problems $\mathrm{P} 2|\mathrm{r}-\mathrm{a}| \Sigma C_{j}$ and $\mathrm{P} 2|\mathrm{r}-\mathrm{a}, \mathrm{s}-\mathrm{p}| \Sigma C_{j}$ are also NP-hard. The following property gives the complexity when the number of machines is arbitrary.

Property 2.1. $\mathrm{P}|\mathrm{r}-\mathrm{a}| \Sigma C_{j}$ and $\mathrm{P}|\mathrm{r}-\mathrm{a}, \mathrm{s}-\mathrm{p}| \Sigma C_{j}$ are NP-hard in the strong sense.

\section{Properties, Bounds, and Algorithm}

This section is comprised of three subsections. We first identify two optimality properties that we employ in a mixed integer linear programming formulation of our problem. Subsection 3.2 contains upper bounds, properties that identify easily solved special cases of our problem, and lower bounds that make use of these properties. The final subsection describes our branch and bound algorithm.

\subsection{Optimality Properties}

The first property refers to the notion of an interior point, which is a point in time $t$ that a job is preempted on machine $i \in[1, m]$ that satisfies $t \in\left(T_{i k-1}+D_{i k-1}, T_{i k}\right)$, i.e., an interior point is a preemption that occurs on a machine while it is available.

Property 3.1. There exists an optimal schedule for $\mathrm{P}|\mathrm{r}-\mathrm{a}| \Sigma C_{j}$ with no interior points, and no preemptions on a machine after the last downtime on the machine.

The next property follows directly from Lee (1996), who shows that the PSPT rule is optimal for $1|\mathrm{r}-\mathrm{a}| \Sigma C_{j}$. 
Property 3.2. In the optimal schedule for $\mathrm{P}|\mathrm{r}-\mathrm{a}| \Sigma C_{j}$, jobs are sequenced according to SPT order on each of the machines.

Properties 3.1 and 3.2 are used in the following formulations of $\mathrm{P}|\mathrm{r}-\mathrm{a}| \Sigma C_{j}$, i.e., the properties allow the optimal schedule on each machine to be determined from the jobs assigned to the machine. We evaluate both formulations in computational experiments that are reported in Section 4.

Decision variables

$$
\begin{aligned}
& x_{j i}= \begin{cases}1 & \text { if job } j \text { is processed on machine } i \\
0 & \text { otherwise }\end{cases} \\
& y_{j k i}= \begin{cases}1 & \text { if job } j \text { is assigned on machine } i \text { and completes after downtime } k \\
0 & \text { otherwise }\end{cases} \\
& C_{j}=\text { completion time of job } j
\end{aligned}
$$

\section{Parameters}

$M_{j i}=$ upper bound on the completion time of job $j$ on machine $i$

$$
=\sum_{\forall k \ni \sum_{l=1}^{k}\left(T_{i l}-T_{i(l-1)}-D_{i(l-1)}\right)<\sum_{l=1}^{j} p_{l}} D_{i k}+\sum_{l=1}^{j} p_{l}
$$

$M_{j}=$ upper bound on the total processing time of jobs already assigned to any machine

$$
=\sum_{l=1}^{j} p_{l}
$$

\section{Model IP 1}

$\operatorname{Min} \sum_{j=1}^{n} C_{j}$

subject to 


$$
\begin{array}{ll}
\sum_{\forall k \ni \sum_{l=1}^{k}\left(T_{i l}-T_{i(l-1)}-D_{i(l-1)}\right)<\sum_{l=1}^{j} p_{l}}\left(T_{i k}-T_{i(k-1)}-D_{i(k-1)}\right) y_{j(k-1) i} \geq \sum_{l=1}^{j} p_{l} x_{l i} & \forall j, \forall i \\
y_{j k i} \geq y_{j(k+1) i} & \forall j, \forall i, \forall k=1, . ., n_{i}-1 \\
C_{j} \geq \sum_{\forall k \ni \sum_{l=1}^{k}\left(T_{i l}-T_{i(l-1)}-D_{i(l-1)}\right)<\sum_{l=1}^{j} p_{l}} y_{j k i} D_{i k}+\sum_{l=1}^{j} p_{l} x_{l i}-M_{j i}\left(1-x_{j i}\right) & \forall j, \forall i \\
\sum_{i=1}^{m} x_{j i}=1 & \forall j \\
x_{j i} \in\{0,1\} & \forall j, \forall i \\
y_{j k i} \in\{0,1\} & \forall j, \forall i, \forall k=1, . ., n_{i}
\end{array}
$$

Constraints (3.2) - (3.3) ensure the proper values of $y_{j k i}$. The inequality that determines the $k$ values in constraint (3.2) (i.e., $\left.\sum_{l=1}^{k}\left(T_{i l}-T_{i(l-1)}-D_{i(l-1)}\right)<\sum_{l=1}^{j} p_{l}\right)$ eliminates downtimes that must occur after job $j$ on machine $i$ in an optimal schedule, and thus removes unnecessary columns and rows from the IP formulation. Constraint (3.4) calculates the completion time of each job based on the job-to-machine assignments defined through the values of $x_{j i}$. Constraint (3.5) guarantees that each job is assigned to a machine, and (3.6) - (3.7) are integrality constraints. An alternative formulation is given below.

Model IP 2

$\operatorname{Min} \sum_{j=1}^{n} C_{j}$

subject to

$$
\sum_{l=1}^{j} p_{l} x_{l i}-\sum_{l=1}^{k}\left(T_{i l}-T_{i(l-1)}-D_{i(l-1)}\right) \leq M_{j} y_{j k i} \quad \forall j, \forall i, \forall k \ni \sum_{l=1}^{k}\left(T_{i l}-T_{i(l-1)}-D_{i(l-1)}\right)<\sum_{l=1}^{j} p_{l}
$$




$$
\begin{array}{lc}
C_{j} \geq \sum_{\forall k \jmath \sum_{l=1}^{k}\left(T_{i l}-T_{i(l-1)}-D_{i(l-1)}\right)<\sum_{l=1}^{j} p_{l}} y_{j k i} D_{i k}+\sum_{l=1}^{j} p_{l} x_{l i}-M_{j i}\left(1-x_{j i}\right) & \forall j, \forall i \\
\sum_{i=1}^{m} x_{j i}=1 & \forall j \\
x_{j i} \in\{0,1\} & \forall j, \forall i \\
y_{j k i} \in\{0,1\} & \forall j, \forall i, \forall k \ni \sum_{l=1}^{j} p_{l}>\sum_{l=1}^{k}\left(T_{i l}-T_{i(l-1)}-D_{i(l-1)}\right)
\end{array}
$$

Constraints (3.8) - (3.9) play the same role (3.2) - (3.4) in model $I P_{1}$. Constraint (3.10) is similar to (3.7) in model $I P_{1}$, though the range of $k$ values is more limited.

\subsection{Upper and Lower Bounds}

In this subsection we propose upper and lower bounds. For upper bounds, we propose two construction type heuristic procedures and an improvement type heuristic to find an initial feasible solution for our implicit enumeration procedure.

Construction Heuristic $1\left(C U B_{1}\right)$ : This heuristic is an adaptation of the PSPT rule proposed by Lee (1996) for the single machine problem. Jobs are assigned to the first available machine in SPT order. If a job does not complete prior to a downtime, it is preempted and is scheduled to resume processing after the downtime.

Construction Heuristic $2\left(C U B_{2}\right)$ : This heuristic is similar to $C U B_{1}$ in that it schedules jobs in SPT order. However, rather than scheduling a job on the first available machine, the job is scheduled on the machine where it will complete earliest. Due to machine downtimes, the first available machine can be different than the machine where the job will complete earliest (e.g., $C U B_{1}$ and $C U B_{2}$ return the same schedule if there are no downtimes or if downtimes are synchronous-periodic, but the schedules can differ dramatically in the asynchronous case). 
Improvement Heuristic (IUB): This heuristic begins with the best schedule from $C U B_{1}$ and $C U B_{2}$. It evaluates the interchange between any two jobs assigned to two different machines, and restarts when a better schedule is found. The procedure terminates when no interchange can improve the schedule.

We identify two lower bounds $-L B_{1}$ and $L B_{2}-$ which we incorporate into our branch and bound algorithm. For $L B_{1}$, we identify a polynomial time algorithm for $\mathrm{P} \mid \mathrm{r}-\mathrm{a}$,split $\mid \Sigma C_{j}$, which is a relaxation of $\mathrm{P}|\mathrm{r}-\mathrm{a}| \Sigma C_{j}$ where jobs are allowed to be arbitrarily split into subjobs that can be processed simultaneously on multiple machines.

In terms of the notation introduced in Section 2, an instance of $\mathrm{P} \mid \mathrm{r}-\mathrm{a}$,split $\mid \Sigma C_{j}$ can be expressed as $I_{1}=\left\{p_{1}, p_{2}, \ldots, p_{n_{s}} m,\left(T_{1,1}, D_{1,1}\right),\left(T_{1,2}, D_{1,2}\right), \ldots,\left(T_{1, n_{1}}, D_{1, n_{1}}\right) ; \ldots ;\left(T_{m, 1}, D_{m, 1}\right),\left(T_{m, 2}\right.\right.$ $\left.\left.D_{m, 2}\right), \ldots,\left(T_{m, n_{m}}, D_{m, n_{m}}\right)\right\}$. For the purposes of specifying our algorithm for P|r-a,split| $\Sigma C_{j}$, we express the instance as $I_{2}=\left\{p_{1}, p_{2}, \ldots, p_{n_{s}}\left(T_{1}, M_{1}\right),\left(T_{2}, M_{2}\right), \ldots,\left(T_{N}, M_{N}\right)\right\}$ where $T_{k}$ is a point in time when $M_{k}$ machines become available for processing. For example, if $I_{1}=\{2,3,5,7,2$, (3, 2); $(\varnothing, \varnothing)\}$ (i.e., 4 jobs, 2 machines, machine 1 is down for 2 periods beginning at time 3), then $I_{2}=\{2,3,5,7,(0,2),(3,1),(5,2)\}$ (i.e., 4 jobs, 2 machines available at time 0,1 machine available at time 3, 2 machines available at time 5).

Note that in contrast to $I_{1}$, instance $I_{2}$ does not keep track of which machine is down. However, for problem $\mathrm{P} \mid \mathrm{r}-\mathrm{a}$,split $\mid \Sigma C_{j}$, there is no need to keep track of which machines are down because, as shown below in Algorithm $A_{1}$ and Property 3.3, each job is processed on all available machines simultaneously until complete in an optimal schedule. In short, the format of $I_{2}$ allows for a simpler specification of an optimal solution algorithm for $\mathrm{P} \mid \mathrm{r}-\mathrm{a}$,split $\mid \Sigma C_{j}$. Consider the following algorithm applied to instance $I_{2}$. 


\section{Algorithm $A_{1}$ :}

1. Sort the jobs from smallest-to-largest processing time and, if necessary, re-index such that $p_{1}$ $\leq p_{2} \leq \ldots \leq p_{n}$.

2. Set $i=j=1, t=T_{1}, T_{N+1}=\Sigma p_{j}$.

3. If $t+p_{j} / M_{i}<T_{i+1}$, then $t=t+p_{j} / M_{i}, C_{j}=t, j=j+1$; otherwise $p_{j}=p_{j}-M_{i}\left(T_{i+1}-t\right), t=T_{i+1}, i$ $=i+1$.

4. If $j \leq n$, then go to step 3 .

5. Return $\Sigma C_{j}$

For example, if $I_{2}=\{2,3,5,7,(0,2),(3,1),(5,2)\}$, then applying $A_{1}$ to $I_{2}$ yields $C_{1}=2 / 2$ $=1, C_{2}=1+3 / 2=2.5, C_{3}=2.5+1 / 2+2 / 1+2 / 2=6, C_{4}=6+7 / 2=9.5$, and $\Sigma C_{j}=1+2.5+6$ $+9.5=19$.

Property 3.3. $A_{1}$ is optimal for $\mathrm{P}|\mathrm{r}-\mathrm{a}, \mathrm{split}| \Sigma C_{j}$.

Splitting Bound $\left(\boldsymbol{L} \boldsymbol{B}_{1}\right)$ : This lower bound transforms an instance of $\mathrm{P}|\mathrm{r}-\mathrm{a}| \Sigma C_{j}$ into the corresponding instance of $\mathrm{P}|\mathrm{r}-\mathrm{a}, \mathrm{split}| \Sigma C_{j}$ and then applies $A_{1}$.

$L B_{1}$ is a lower bound for $\mathrm{P}|\mathrm{r}-\mathrm{a}| \Sigma C_{j}$ because $\mathrm{P} \mid \mathrm{r}-\mathrm{a}$,split| $\mid \Sigma C_{j}$ is a relaxation of $\mathrm{P}|\mathrm{r}-\mathrm{a}| \Sigma C_{j}$ (i.e., constraint requiring that a job be processed in its entirety on a single machine is relaxed).

Note that $L B_{1}$ is valid for any number of machines. $L B_{2}$, on the other hand, is limited to the case of $m=2$ and is based on the following two properties. In particular, Property 3.4 exposes an insight that is relevant for a new type of bound-a shifting bound - that has not previously appeared in the literature to our knowledge. The property, in combination with Property 3.5, shows how we can transform an SPT schedule that has preemptions into a schedule without preemptions to yield a lower bound on the optimum of the original problem.

Property 3.4. If an SPT schedule applied to an instance of $\mathrm{P} 2|\mathrm{r}-\mathrm{a}| \Sigma C_{j}$ results in no preemptions, then the schedule is optimal. 
Property 3.5. For $\mathrm{P}|\mathrm{r}-\mathrm{a}| \Sigma C_{j}$, the optimal total completion time is nonincreasing as one or more downtimes are shifted to the right.

Shifting Bound $\left(\boldsymbol{L} \boldsymbol{B}_{2}\right)$ : This lower bound applies the SPT rule. When a job will not complete prior to a downtime, the downtime is shifted to the right to the point in time when the job completes processing. If the shifting of a downtime causes the end of the downtime to overlap with a subsequent downtime, then the subsequent downtime is shifted to the right by the amount necessary to avoid overlap.

\subsection{Branch and Bound Algorithm}

In our branch and bound (B\&B) tree, each level corresponds to the next position on the earliest available machine, and the decision represented by a node is the job to be assigned. We also consider the case of closing down the earliest available machine, i.e., assigning no more jobs to that specific machine. Our $\mathrm{B} \& \mathrm{~B}$ tree has at most $n+m-1$ (i.e., $n$ for the number of jobs, $m$ for closing machines from future assignments, and 1 for the root node), and at each level it has at most $n+1$ nodes.

We use a depth-first search strategy due to its efficient use of memory. We select the node with the smallest lower bound. In addition, we utilize Property 3.2 in construction our B\&B tree, and avoid any non-SPT sequencing in the partial schedules. In particular, from Property 3.2 we know that jobs are scheduled in increasing order of processing times on each machine in an optimal solution. Thus, by branching on the earliest available machine, we avoid creating child nodes for which jobs having smaller processing times than the last assigned job are scheduled.

We implement the following algorithm using either $L B_{1}$ or $L B_{2}$ as the lower bound (see the appendix for an example of the algorithm applied to a small instance). When implemented using $L B_{1}$, we refer to the algorithm as $B \& B_{1}$; when implemented using $L B_{2}$, we refer to the 
algorithm as $B \& B_{2}$. Note that $B \& B_{1}$ can be applied to instances with any number of machines, whereas $B \& B_{2}$ is limited to instances with two machines.

\section{Algorithm $B \& B_{i}$ :}

1. Utilize the two construction heuristics, to initialize the initial feasible solution: $U B=$ $\min \left\{C U B_{1}, C U B_{2}\right\}$

2. Utilize the improvement heuristic on the initial feasible solution: $U B=I U B$

3. Initialize the stack with the root node: $S T=\{(0,0)\}$

4. Find the earliest available machine, $\min _{m}$, and create children nodes by assigning jobs obeying the SPT rule: $J \rightarrow \min _{m}$

5. Evaluate the children nodes using lower bound, fathom if $L B_{i} \geq U B$; if $L B_{i}$ schedule is feasible and $L B_{i}<U B$, then $U B=L B_{i}$ and fathom

6. Create a child node for closing down the machine, $J \rightarrow$ closed, and evaluate the child node using lower bound, fathom if $L B_{i} \geq U B$; if $L B_{i}$ schedule is feasible and $L B_{i}<U B$, then $U B=$ $L B_{i}$ and fathom

7. Add all non-fathomed jobs in decreasing order of their lower bound values to the stack

8. If $S T \neq \varnothing$, go to step 4

9. Return the optimal schedule

Note that for a problem with synchronous downtimes there are at least two alternative optimal schedules (e.g., two ways to label the two machines). The number of alternative optimal schedules can be larger due to multiple occurrences of points in time when all machines become simultaneously available (and simultaneously unavailable). These alternative optimal solutions could significantly increase the number of nodes evaluated. Since our aim is to identify an optimal solution instead of identifying set of alternate optimal solutions, we modify our B\&B algorithm to consider only one specific assignment of jobs to machines which become simultaneously available. We assign jobs with an increasing order of the job index values to machines with an increasing order of the machine index values. For instance, for a two machine 
problem, if job 1 is assigned to machine 1 , then we eliminate the alternate optimal solution of job 1 assigned to machine 2 .

\section{Computational Experiments}

We conduct computational experiments to assess the efficiency of our algorithms. We generate random problem instances having $n=10,15,20,25,30$, and 35 jobs and two machines. The job processing times are drawn from two discrete uniform distributions with ranges $[1,30]$ and $[1,100]$, representing different levels of variation in processing times. We work with the two different cases of planned downtimes — synchronous-periodic and asynchronous.

Synchronous-periodic downtimes

The expected number of downtimes scheduled on each machine is set at three different values: 1,3 and 5 . We use the following formula to compute the amount of time between downtimes.

$$
\text { Periodic uptime }=\frac{\sum_{j} p_{j}}{m(F+1)} \quad F=1,3,5
$$

The downtime duration is set at two levels-long, which is the average processing time, and short, which is one-half of the average processing time, i.e.,

Periodic downtime $(\mathrm{L})=\frac{\sum_{j} p_{j}}{n}$

Periodic downtime $(\mathrm{S})=\frac{\sum_{j} p_{j}}{2 n}$ 
Asynchronous downtimes

For the asynchronous case, the uptime and downtime durations are randomly generated from a discrete uniform (DU) distribution. The expected uptime and downtime values match the deterministic values in the synchronous-periodic case.

Random uptime $\sim$ DU $\left[\frac{1}{2} \frac{n E\left[p_{j}\right]}{m(F+1)}, \frac{3}{2} \frac{n E\left[p_{j}\right]}{m(F+1)}\right] \quad F=1,3,5$

Random downtime (L) $\sim \mathrm{DU}\left[\frac{1}{2} E\left[p_{j}\right], \frac{3}{2} E\left[p_{j}\right]\right]$

Random downtime $(\mathrm{S}) \sim \mathrm{DU}\left[\frac{1}{4} E\left[p_{j}\right], \frac{3}{4} E\left[p_{j}\right]\right]$

We replicate each setting ten times, and conduct the experiment on a PC with Intel Pentium $42.8 \mathrm{GHz}$ processor and $1 \mathrm{~GB}$ of RAM running under Windows XP SP2. We implemented our B\&B algorithm in C, compiled with GCC 3.4.5, and utilized Borland C++BuilderX 1.0 as the development environment.

To help assess the merits of our B\&B algorithm relative to commercial software, we solved long duration instances with CPLEX 10 using formulations $I P_{1}$ and $I P_{2}$. The following tables report average CPU time in seconds of our B\&B algorithms and CPLEX with the two IP formulations. An asterisk in a cell indicates that at least one of the ten instances was not solved within 7,200 seconds, or two hours. The tables terminate at $n=25$ and $F=1$, because both $I P_{1}$ and $I P_{2}$ did not return a solution within two hours for instances with larger values of $n$ and/or $F$.

\section{INSERT TABLES 4.1 AND 4.2 HERE}

The results show that both $\mathrm{B} \& \mathrm{~B}$ algorithms are superior to CPLEX with either IP formulation. The difference in performance increases in instance size, which is not surprising 
because the number of binary variables and constraints increase with the number of jobs and downtimes.

We see that $I P_{2}$ is superior to $I P_{1}$ with the difference increasing in instance size. This difference can at least partially be attributed to the efficiency of model formulations in terms of the number of integer and continuous decision variables and constraints used, i.e., $I P_{2}$ has fewer decision variables and constraints.

The following two tables provide details on the performance of the B\&B algorithms. The tables report average number of nodes and CPU time in seconds. An asterisk in a cell indicates that at least one of the ten instances was not solved within 10,800 seconds, or three hours.

\section{INSERT TABLES 4.3 AND 4.4 HERE}

The results show that $B \& B_{1}$, which is based on the splitting bound, generally improves as the total downtime within the planning horizon increases. An increase in the downtime frequency and / or duration reduces the number of job-splitting possibilities, and thus results in a tighter lower bound. On the other hand, $B \& B_{2}$, which is based on the shifting bound, generally degrades as the total downtime within the planning horizon increases. This is because a reduction in the downtime frequency and / or duration lowers the necessity of utilizing right-shifts in downtimes, thereby tightening the lower bound.

In terms of comparative $\mathrm{B} \& \mathrm{~B}$ performance, we find that that $B \& B_{2}$ generally outperforms $B \& B_{1}$ (i.e., the shifting bound is generally more effective than the splitting bound). An exception occurs with instances having the highest downtime duration and frequency.

We also ran numerical experiments that included a version of the $\mathrm{B} \& \mathrm{~B}$ with $L B=$ $\max \left\{L B_{1}, L B_{2}\right\}$. We found that this $\mathrm{B} \& \mathrm{~B}$ generally performed worse than the better of $B \& B_{1}$ and $B \& \mathrm{~B}_{2}$, and consequently we did not report the results in the tables. The reason for the result is 
that the types of instances where $L B_{1}$ or $L B_{2}$ tends to dominate are quite distinct; $B \& B_{1}$ (based on $L B_{1}$ ) dominates when downtime duration and frequency are high, otherwise $B \& B_{2}$ (based on $\left.L B_{2}\right)$ dominates. Computing both bounds in a $\mathrm{B} \& \mathrm{~B}$ adds overhead with minimal reduction in the $\mathrm{B} \& \mathrm{~B}$ tree.

The results show that asynchronous problems are generally more difficult to solve than synchronous-periodic problems. This can be attributed to our mechanisms designed to avoid generating and evaluating multiple optimal schedules. These mechanisms only go into effect when downtimes are synchronous and periodic. We also see that, for instances with synchronous-periodic downtimes, the $\mathrm{B} \& \mathrm{~B}$ algorithms perform notably worse on the problem instances with job processing time distributions DU[1,100] than with DU[1,30]. Job processing time variation has little effect on B\&B performance with downtimes are asynchronous. When downtimes are synchronous and periodic, there are blocks of time when all machines are available, and an important factor influencing the objective function is scheduling a large number of jobs to complete during time blocks when machines are running. As variation in job processing time increases, the required effort to find a good "fitting" of jobs into these time blocks increases, and thus B\&B performance degrades.

In order to assess the quality of the three upper bounds introduced in Section 3.2, we computed the percentage increase above the optimum. Tables 4.5 and 4.6 report the average percentage increase and the maximum percentage increase for each heuristic. The heuristic CPU times were negligible (i.e., always less than 0.005 seconds).

\section{INSERT TABLES 4.5 AND 4.6 HERE}

The results show that the construction heuristics $\left(C U B_{1}\right.$ and $\left.C U B_{2}\right)$ are within $2 \%$ of the optimum on average, and do not exceed $20 \%$ in any single problem instance. (Note that $C U B_{1}$ 
and $C U B_{2}$ generate the same schedule for the synchronous-periodic problems because all machines have the same downtime schedule.) The improvement heuristic (IUB), which attempts to improve upon the best schedule from $C U B_{1}$ and $C U B_{2}$, reduces the gap by about a factor of five on average, and the maximum gap over all instances is less than $7 \%$.

\section{Conclusion}

We study the two identical parallel machine scheduling problem with planned downtimes and resumable jobs. We consider two practical variations of this problem with different types of planned downtimes - one where downtimes are synchronous and periodic, which can occur in environments operated with multiple shifts, and the other where downtimes are asynchronous, which represents a general case.

We prove that the problems are NP-hard. We propose two construction heuristics and one improvement heuristic, and we propose two lower bounds based on splitting and shifting relaxations. We develop a $B \& B$ algorithm based on these bounds and several optimality properties. We generate a set of problem instances to evaluate our B\&B algorithms, and we summarize structural characteristics of the performance. The results of our experiments show that our B\&B is far superior to IP formulations that are solved using CPLEX 10, one of the best available commercial packages. Our B\&B algorithm is capable of solving problems of sizes up to 35 jobs in certain settings.

We have identified properties of an optimal schedule, and we have identified two variations of the problem that can be solved in polynomial time (which underlie our lower bounds). Future research should identify analogous and / or new properties for problems with preempt-repeat or preempt-resume with fixed setup. Other extensions that should be considered include models of multistage networks and models where the speed of a processor can be 
between the extremes of its nominal speed (e.g., machine is available) and zero (e.g., machine is unavailable). Both these features reflect realities of practice. Finally, as work progresses on methods for generating schedules when downtimes / slowdowns are known, it will be worthwhile to explore the application of these methods to problems where downtimes / slowdowns are random variables, and more generally, to the question how supply chain design choices affect the sensitivity of system performance to disruptions.

\section{Appendix}

Proof of Property 2.1. Let $P_{2}$ denote the recognition version of $\mathrm{P}|\mathrm{r}-\mathrm{a}| \Sigma C_{j}$ (or $\left.\mathrm{P}|\mathrm{r}-\mathrm{a}, \mathrm{s}-\mathrm{p}| \Sigma C_{j}\right)$ An instance of $P_{2}$ can be expressed as $I_{2}=\left\{p_{1}, p_{2}, \ldots, p_{n_{s}} m,\left(T_{1,1}, D_{1,1}\right),\left(T_{1,2}, D_{1,2}\right), \ldots,\left(T_{1, n_{1}}, D_{1, n_{1}}\right)\right.$, $\left.\ldots,\left(T_{m, 1}, D_{m, 1}\right),\left(T_{m, 2}, D_{m, 2}\right), \ldots,\left(T_{m, n_{m}}, D_{m, n_{m}}\right), K\right\}$. Problem $P_{2}$ asks if there is a schedule $\sigma$ satisfying $\Sigma C_{j}(\sigma) \leq K$.

Let $P_{1}$ denote the recognition version of $\mathrm{P} \| C_{\max }$. An instance of $P_{1}$ can be expressed as $I_{1}$ $=\left\{p_{1}, p_{2}, \ldots, p_{n_{s}} m, T\right\}$. Problem $P_{1}$ asks if there is a schedule $\sigma$ satisfying $C_{j}(\sigma) \leq T$ for all $j$.

We will define a polynomial transformation of $I_{1}$ to $I_{2}$, and show that $I_{1}$ is yes if and only if $I_{2}$ is yes. From the fact that $P_{1}$ is strongly NP-complete (Garey and Johnson (1978)), it follows that $P_{2}$ is strongly NP-complete. This fact combined with the fact that strong NP-completeness of a recognition version of an optimization problem implies that the optimization problem (which is not in the set NP) is strongly NP-hard leads to the desired result.

The polynomial time transformation of an instance of $P_{1}$ into an instance of $P_{2}$ is obtained as follows (prior to the transformation, parameters in instance $I_{1}$ are indexed such that

$$
\begin{aligned}
p_{1} \leq & \left.p_{2} \leq \ldots \leq p_{n}\right): \\
& p_{j}=p_{j} \text { for } j=1,2, \ldots, n=\text { job processing times } \\
& m=m=\text { the number of identical machines }
\end{aligned}
$$


$T_{i, 1}=T$ for $i=1,2, \ldots, m=$ beginning of machine downtimes

$D_{i, 1}=\Sigma j p_{j}$ for $i=1,2, \ldots, m=$ duration of machine downtimes

$K=\Sigma j p_{j}=$ limit on total flowtime

Note that $\Sigma j p_{j}=$ total flowtime when jobs are sequenced on a single machine in order $n$, $n-1, \ldots, 1$. Now observe that for any given schedule $\sigma$, we have $\Sigma C_{j}(\sigma) \leq K$ if and only if $C_{j}(\sigma) \leq$ $T$ for all $j$. The reason is that if any job $j$ completes processing after the planned downtime $T$, then $C_{j}(\sigma)>K$ (and $\Sigma C_{j}(\sigma)>K$ ); in addition, due to the definition of $K$ (i.e., maximum total flowtime of a single machine problem), if all jobs complete before the planned downtime $T$ we can be assured that $\Sigma C_{j}(\sigma) \leq K$. The proof essentially follows from this observation. Formally, if $I_{2}$ is yes, then by definition there exists a schedule $\sigma$ such that $\Sigma C_{j}(\sigma) \leq K$, which implies $C_{j}(\sigma) \leq$ $T$ for all $j$, and $I_{1}$ is yes. Alternatively, if $I_{2}$ is no, then by definition, we have $\Sigma C_{j}(\sigma)>K$ for all schedules, which implies $C_{j}(\sigma)>T$ for some $j$ in all schedules, and $I_{1}$ is no.

Proof of Property 3.1. Preemption is never necessary to improve total flowtime on a machine after the last planned downtime. What remains is to prove that there always exists an optimal schedule with no interior points.

Suppose the property is not true. Then there exists a problem instance where all optimal schedules have interior points. Consider one such optimal schedule. We have some job, say job $k$, that is preempted on machine $l$ at an interior point. Let $k_{1}$ denote the contiguous portion of job $k$ that is preempted at time $t$, which is the $i^{\text {th }}$ interior point of job $k$. Let $k_{2}$ denote the last contiguous portion of job $k$ (i.e., the completion time of portion $k 2$ is the completion time of job $k)$.

We will describe a change to the schedule on machine $l$ that eliminates the $i^{\text {th }}$ interior point of job $k$ without creating any additional interior points and without increasing total 
completion time. This method can be continually repeated until there are no interior points on any machine. The flowtime of the resulting schedule must be the same or better than the optimum, which is a contradiction, and consequently the property must be true.

Note that there is no idle time in the schedule for machine $l$; if there was, jobs could be shifted to the left (preempting around planned downtimes as needed), and flowtime would be reduced. Remove $k_{1}$ from the schedule, shift all jobs between $k_{1}$ and $k_{2}$ to the left as much as possible (preempting at the start of planned downtimes as needed). After this shift, the amount of idle time just prior to $k_{2}$ is exactly equal to the time to process $k_{1}$ (because there was no idle time on the machine in the original schedule). For the last step, place $k_{1}$ in the schedule just before $k_{2}$.

With the above change, we see that the completion times for job $k$ and jobs completing after job $k$ on machine $l$ are unchanged. The completion times of jobs or portions of jobs scheduled between $k_{1}$ and $k_{2}$ cannot increase, and no additional interior points are created. Neither the total flowtime nor the number of interior points after the change can increase.

Proof of Property 3.3. Let $m(t)=$ speed at time $t$ (i.e., units per period). For example, for $I_{2}=\{2$, $3,5,7,(0,2),(3,1),(5,2)\}$, we have

$$
m(t)=\left\{\begin{array}{l}
2, t \in[0,3) \\
1, t \in[3,5) . \\
2, t \geq 5
\end{array} .\right.
$$

Note that $\int_{a}^{b} m(t) d t=$ number of units processed by the machine during the time interval $[a, b]$. Let $p_{j}=$ number of units for job $j$ (i.e., job processing time can be interpreted as a number of units to be produced, where a unit is defined such that it takes 1 period to process on a machine of speed 1). Let $[i]=$ index of the job in the $i^{\text {th }}$ position in a sequence on the machine. Let $C_{j}=$ completion time of job $j$ in some schedule. We have the following relationships: 


$$
\begin{aligned}
& p_{[i]}=\int_{C_{[i-1]}}^{C_{[i]}} m(t) d t \\
& p_{[1]}+p_{[2]}+\ldots+p_{[i]}=\int_{0}^{C_{[i]}} m(t) d t \\
& \Sigma p_{j}=\int_{0}^{C_{[n]}} m(t) d t
\end{aligned}
$$

We can see that $C_{[n]}$ is independent of the sequence, i.e., $C_{[n]}$ is the time point such that area under the function $m(t)$ is exactly equal to $\Sigma p_{j}$.

Applying the above notation, the property can be proved through standard interchange arguments. For example, consider any pair of consecutive jobs that are not in SPT order. By interchanging the jobs we will see that sum of the completion times for the pair of jobs is reduced. Such a result leads to the conclusion that a schedule is optimal if and only if the job sequence is consistent with SPT ordering. Algorithm $A_{1}$ generates a schedule based on SPT ordering, and thus is optimal. We illustrate the impact of a job interchange below.

Suppose jobs $k$ and $j$ are processed consecutively in a schedule, and $p_{k}>p_{j}$, i.e., the jobs are not consistent with SPT ordering. Let $s_{k}$ be the start time of job $k$ in the schedule. We have

$$
p_{k}=\int_{s_{k}}^{C_{k}} m(t) d t \text { and } p_{k}+p_{j}=\int_{s_{k}}^{C_{j}} m(t) d t
$$

Now interchange the jobs, and let the completion times after interchange be denoted as $C_{j}^{\prime}$ and $C_{k}{ }^{\prime}$. We have

$$
p_{j}=\int_{s_{k}}^{C_{j^{\prime}}{ }^{\prime}} m(t) d t \text { and } p_{j}+p_{k}=\int_{s_{k}}^{C_{k}{ }^{\prime}} m(t) d t=\int_{s_{k}}^{C_{j}} m(t) d t .
$$

Since $p_{j}<p_{k}$, it follows that $C_{j}^{\prime}<C_{k}$. Thus,

$$
C_{j}^{\prime}+C_{k}^{\prime}=C_{j}^{\prime}+C_{j}<C_{k}+C_{j},
$$


and completion time is reduced as a result of the interchange.

Proof of Property 3.4. There are $v$ downtimes. Downtime $l$ of duration $d_{l}$ begins at time $t_{l}$, and downtimes are indexed in increasing order of start times (i.e., $t_{1} \leq t_{2} \leq \ldots \leq t_{v}$ ). Let $W_{i}^{*}$ and $W_{i}^{\mathrm{S}}$ denote the time that the last job completes processing on machine $i$ in an optimal and SPT schedule, respectively. We use $s_{j}{ }^{*}$ and $s_{j}$ S to denote the start times of job $j$ in an optimal and SPT schedule, respectively.

Jobs are indexed in SPT order. Let $I_{k, l}$ be instance $I$ with the $k$ longest jobs and the last $l$ downtimes removed, e.g., $I_{1,0}=\Lambda\left\{p_{n}\right\}, I_{0,1}=\Lambda\left\{t_{v}, d_{v}\right\}, I_{1,1}=\Lambda\left\{p_{n}, t_{v}, d_{v}\right\}, I_{2,1}=\Lambda\left\{p_{n-1}, p_{n}, t_{v}, d_{v}\right\}$, etc. An instance $I$ is minimal if

1. $\Sigma C_{j}^{\mathrm{S}}(I)>\Sigma C_{j}^{*}(I)$

2. $\Sigma C_{j}^{\mathrm{S}}\left(I_{k, 0}\right)=\Sigma C_{j}^{*}\left(I_{k, 0}\right)$ for all $k \geq 1$

3. $\Sigma C_{j}^{\mathrm{S}}\left(I_{0, l}\right)=\Sigma C_{j}^{*}\left(I_{0, l}\right)$ for all $l \geq 1$

Suppose that the property is not true. Then there must exist a minimal instance $I$, i.e., we have

$$
\Sigma C_{j}^{\mathrm{S}}>\Sigma C_{j}^{*}
$$

(For notational simplicity, with the exception of instances that differ from $I$, we exclude the argument $I$ in $C_{j}^{\mathrm{S}}(I)$ and $C_{j}^{*}(I)$.) Job $n$ is the makespan job in the SPT schedule. Let $\eta$ be the makespan job in the optimal schedule.

Case 1: job $n-1$ is scheduled before job $n$ on the same machine in the SPT schedule

Since jobs $n-1$ and $n$ are consecutively scheduled on a machine in the SPT schedule, it must be that the other machine, say machine 1, is down when job $n$ is processing, i.e., $C_{n-1}{ }^{\mathrm{S}}=s_{n}{ }^{\mathrm{S}} \leq t_{\kappa}+d_{\kappa}$ for some $\kappa \leq v$. The following figure shows two examples of a possible SPT schedule.

Downtime $\kappa \neq v$ 
M1: $\quad \ldots \quad|--(j \circ b \quad j 1)--| x \times x(\kappa) \operatorname{xxxx}$

M2: $\quad \ldots \quad|--(j \circ b n-1)--| x(v) x|----j o b n----|$

Downtime $\kappa=v$

M1: $\quad . . \quad|--(j \circ b \quad j 1)--| \operatorname{xxx}(\kappa) \operatorname{xxxx}$

M2: $\quad . . \quad|--(j \circ b n-1)--|-----j \circ b \quad n-----1$

We consider three possible sub-cases, and for each we show that the SPT schedule must be optimal. We say that a downtime on a machine is spanned if a job completes processing after the downtime on the machine.

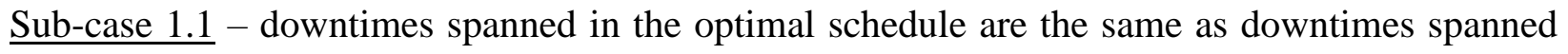
in the SPT schedule. The makespan of the SPT schedule is $C_{n}^{\mathrm{S}}$. Since all downtimes except downtime $\kappa$ are spanned in both schedules, it must be that $C_{\eta}{ }^{*}=C_{n} \mathrm{~S}\left(=\Sigma p_{j}+\Sigma d_{k}-d_{\kappa}-t_{\kappa}\right)$, and we have

$$
\Sigma C_{j}^{\mathrm{S}}=\Sigma_{j<n} C_{j}^{\mathrm{S}}+C_{n}^{\mathrm{S}}=\Sigma C_{j}^{*}\left(I_{1,0}\right)+C_{n}^{\mathrm{S}}=\Sigma C_{j}^{*}\left(I_{1,0}\right)+C_{\eta}^{*} \leq \Sigma_{j \neq \eta} C_{j}^{*}+C_{\eta}^{*}=\Sigma C_{j}^{*}
$$

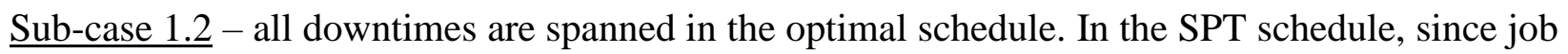
$n$ begins no later than $t_{\kappa}+d_{\kappa}$ and job $n-1$ finishes no sooner than $t_{\kappa}$, it must be that $C_{n-1}{ }^{\mathrm{S}}+C_{n}{ }^{\mathrm{S}} \leq$ $\Sigma p_{j}+\Sigma d_{k}=W_{1}^{*}+W_{2}^{*}$. Therefore,

$$
\Sigma C_{j}^{\mathrm{S}}=\sum_{j<\mathrm{n}-1} \mathrm{C}_{j}^{\mathrm{S}}+C_{n-1} \mathrm{~S}+C_{n}^{\mathrm{S}} \leq \Sigma C_{j}^{*}\left(I_{2,0}\right)+W_{1}^{*}+W_{2}^{*} \leq \Sigma C_{j}^{*}
$$

$\underline{\text { Sub-case } 1.3}$ - the optimal schedule spans downtime $\kappa$, but does not span downtime $v$. The following figure illustrates possible SPT and optimal schedules that correspond to this sub-case.

$\underline{\text { SPT schedule }}$

M1: $\quad$... $|--(j \circ b \quad j 1)--| \mathrm{xxx}(\kappa) \times \mathrm{xxx}$

M2: $\quad \ldots \quad|--(j o b n-1)--| x(v) \times|----j o b n----|$

Candidate optimal schedule

M1 : $\quad \ldots \quad|-(j o b \quad j 2)-| \operatorname{xxx}(\kappa) \operatorname{xxxx} \mid---j o b \quad \eta^{----\mid}$

M2: $\quad \ldots \quad|--(j \circ b \quad j 3)---| x(v) x$ 
Note that any downtimes not spanned in the optimal schedule (e.g., $\kappa+1, \kappa+2, \ldots, v)$ must all start after downtime $\kappa$ begins and complete before downtime $\kappa$ finishes. Consequently, we can simplify our explanation by combining all these downtimes into a single downtime $v$. In the optimal schedule there are no jobs scheduled after downtime $v$ on machine 2 . Therefore, $C_{\eta}{ }^{*}$ $=\Sigma p_{j}+\Sigma d_{k}-d_{v}-t_{v}$. Similarly, all downtimes except downtime $\kappa$ are spanned in the SPT schedule, and thus $C_{n}{ }^{\mathrm{S}}=\Sigma p_{j}+\Sigma d_{k}-d_{\kappa}-t_{\kappa}$. Since $t_{\kappa}+d_{\kappa} \geq t_{v}+d_{\nu}$, we have $C_{\eta}{ }^{*}=\Sigma p_{j}+\Sigma d_{k}-$ $\left(d_{v}+t_{v}\right) \geq \Sigma p_{j}+\Sigma d_{k}-\left(d_{\kappa}+t_{\kappa}\right)=C_{n}^{\mathrm{S}}$ and

$$
\Sigma C_{j}^{\mathrm{S}}=\Sigma_{j<n} C_{j}^{\mathrm{S}}+C_{n}^{\mathrm{S}}=\Sigma C_{j}^{*}\left(I_{1,0}\right)+C_{n}^{\mathrm{S}} \leq \Sigma C_{j}^{*}\left(I_{1,0}\right)+C_{\eta}^{*} \leq \Sigma_{j \neq \eta} C_{j}^{*}+C_{\eta}{ }^{*}=\Sigma C_{j}^{*}
$$

Consequently there is no instance satisfying Case 1 with $\Sigma C_{j}^{\mathrm{S}}>\Sigma C_{j}^{*}$.

Case 2: jobs $n-1$ and $n$ are scheduled last on each machine in the SPT schedule

Let $j i$ denote the job that is scheduled last on machine $i$ in an optimal schedule, and let $J=\{j 1$, $j 2$ \}. Observe that

$$
\begin{aligned}
& \Sigma C_{j}^{\mathrm{S}}=\sum_{j \leq n-2} C_{j}^{\mathrm{S}}+W_{1}{ }^{\mathrm{S}}+W_{2}{ }^{\mathrm{S}}=\Sigma C_{j}{ }^{*}\left(I_{2,0}\right)+W_{1}{ }^{\mathrm{S}}+W_{2}{ }^{\mathrm{S}} \\
& \Sigma C_{j}^{*}=\Sigma_{j \notin J} C_{j}^{*}+W_{1}{ }^{*}+W_{2}{ }^{*} \geq \Sigma C_{j}^{*}\left(I_{2,0}\right)+W_{1}{ }^{*}+W_{2}{ }^{*}
\end{aligned}
$$

There are two possibilities for the form of the SPT schedule: (1) jobs $n-1$ and $n$ both begin after all downtimes on their machines, (2) job $n-1$ completes prior to a downtime on its machine and job $n$ begins after all downtimes on its machine. (Job $n$, which is the makespan job, cannot complete before a downtime on its machine; if it did, then the instance would not be minimal.)

In the case of the first possibility, we have

$$
\begin{gathered}
\Sigma W_{i}^{\mathrm{S}}=\Sigma p_{j}+\Sigma d_{k} \text { and } \\
\max _{k}\left\{t_{k}+d_{k}\right\} \leq s_{n} \mathrm{~S} \leq\left(\Sigma p_{j}+\Sigma d_{k}-p_{n}\right) / 2 \\
\Rightarrow \Sigma p_{j}-p_{n}+\Sigma d_{k} \geq 2 \max _{k}\left\{t_{k}+d_{k}\right\}
\end{gathered}
$$




$$
\begin{aligned}
& \Rightarrow \Sigma p_{j}-p_{\eta}+\Sigma d_{k} \geq 2 \max _{k}\left\{t_{k}+d_{k}\right\} \\
& \Rightarrow C_{l}^{*} \geq \max _{k}\left\{t_{k}+d_{k}\right\} \text { for some } l \neq \eta
\end{aligned}
$$

Therefore all downtimes are spanned in an optimal schedule (e.g., if job $l$ is the last job on the machine without downtime $\kappa=\operatorname{argmax}_{k}\left\{t_{k}+d_{k}\right\}$, then job $\eta$ is scheduled after downtime $\kappa$ in an optimal schedule; alternatively, if job $l$ is the last job on the machine with downtime $\kappa$ and thus spans downtime $\kappa$, then job $\eta$ is last on the other machine after all downtimes). Since all downtimes are spanned in an optimal schedule, we have

$$
W_{1}^{*}+W_{2}^{*}=W_{1}^{\mathrm{S}}+W_{2}^{\mathrm{S}}=\Sigma p_{j}+\Sigma d_{k}
$$

However, (A.1) - (A.3) imply $\Sigma C_{j}^{\mathrm{S}} \leq \Sigma C_{j}^{*}$.

In the case of the second possibility, let $\kappa$ be the index of the downtime scheduled after job $n-1$, i.e., $\kappa=\operatorname{argmax}_{k}\left\{t_{k}+d_{k}\right\}$. We have

$$
\begin{aligned}
& \sum W_{i}^{\mathrm{S}}=\Sigma p_{j}+\Sigma d_{k}-d_{\kappa} \text { and } \\
& \max _{k \neq \kappa}\left\{t_{k}+d_{k}\right\} \leq s_{n} \mathrm{~S} \leq\left(\Sigma p_{j}+\sum_{k \neq \kappa} d_{k}-p_{n}\right) / 2 \\
\Rightarrow & \Sigma p_{j}-p_{n}+\Sigma_{k \neq \kappa} d_{k} \geq 2 \max _{k \neq \kappa}\left\{t_{k}+d_{k}\right\} \\
\Rightarrow & \Sigma p_{j}-p_{\eta}+\sum_{k \neq \kappa} d_{k} \geq 2 \max _{k \neq \kappa}\left\{t_{k}+d_{k}\right\} \\
\Rightarrow & C_{l}^{*} \geq \max _{k \neq \kappa}\left\{t_{k}+d_{k}\right\} \text { for some } l \neq \eta
\end{aligned}
$$

Therefore all downtimes, with the possible exception of downtime $\kappa$, are spanned in an optimal schedule. Thus we have

$$
W_{1}^{*}+W_{2}^{*} \geq W_{1}^{\mathrm{S}}+W_{2}^{\mathrm{S}}=\Sigma p_{j}+\Sigma d_{k}-d_{\kappa}
$$

However, (A.1), (A.2), and (A.4) imply $\Sigma C_{j}^{\mathrm{S}} \leq \Sigma C_{j}^{*}$. In conclusion, the preceding results contradict our supposition that the property is false. 
Proof of Property 3.5. Let $\sigma^{*}(I)$ denote an optimal schedule for instance $I$ of the problem and let $M_{i}\left(\sigma^{*}(I)\right)$ denote the ordered set of job indices assigned to machine $i \in\{1,2, \ldots, m\}$ in schedule $\sigma^{*}(I)$

While maintaining the job-to-machine assignments and job sequences on each machine, consider the impact of shifting one or more downtimes to the right, which yields new instance $I^{\prime}$. Let $\sigma^{\prime}\left(I^{\prime}\right)$ denote the schedule (that is derived from $\sigma^{*}(I)$ ). By Property A.1 (below) we have

$$
\sum_{j \in M_{i}\left(\sigma^{*}(I)\right)} C_{j}\left(\sigma^{*}(I)\right) \geq \sum_{j \in M_{i}\left(\sigma^{*}(I)\right)} C_{j}\left(\sigma^{\prime}\left(I^{\prime}\right)\right) \forall i \in\{1,2, \ldots, m\},
$$

which implies

$$
\sum_{j=1}^{n} C_{j}\left(\sigma^{*}(I)\right) \geq \sum_{j=1}^{n} C_{j}\left(\sigma^{\prime}\left(I^{\prime}\right)\right) \geq \sum_{j=1}^{n} C_{j}\left(\sigma^{*}\left(I^{\prime}\right)\right) \square
$$

Property A.1. For $1|\mathrm{r}-\mathrm{a}| \Sigma C_{j}$, the optimal total completion time is nonincreasing as one or more downtimes are shifted to the right.

Proof. Consider an optimal schedule for an instance of the problem, which is optimal. Let job $j$ be the first job that completes after a downtime with completion time $C_{j}$, and consider the impact of shifting the downtime to the right. There is no change in job completion times until the downtime is shifted to the point where job $j$ completes before the downtime, in which case new completion time of job $j$, denoted $C_{j}^{\prime}$, is

$$
C_{j}^{\prime}=C_{j}-D
$$

where $D$ is the duration of the shifting downtime. No other job completion times are changed. Therefore right-shifting a downtime cannot increase the optimal completion time.

Illustration of B\&B Logic. Consider the problem instance $I=\{1,2,3,4,2,(2,3) ;(4,1)\}$, i.e., job processing times are 1, 2, 3, 4, there are 2 machines, machine 1 is down for 3 periods 
beginning at time 2 , and machine 2 is down for 1 period beginning at time 1 . For this instance, the logic and results of heuristics $C U B_{1}$ and $C U B_{2}$ are illustrated below.

$C U B_{1}$

$\begin{array}{lrrr}\boldsymbol{a}_{1}=\mathbf{0}, a_{2}=0 & \mathrm{~J} 1 \rightarrow \mathrm{M} 1 & C_{1}=1 & \sum C_{i}=1 \\ a_{1}=1, \boldsymbol{a}_{2}=\mathbf{0} & \mathrm{J} 2 \rightarrow \mathrm{M} 2 & C_{2}=2 & \sum C_{i}=3 \\ \boldsymbol{a}_{1}=\mathbf{1}, a_{2}=2 & \mathrm{~J} 3 \rightarrow \mathrm{M} 1 & C_{3}=7 & \sum C_{i}=10 \\ a_{1}=7, \boldsymbol{a}_{2}=\mathbf{2} & \mathrm{J} 4 \rightarrow \mathrm{M} 2 & C_{4}=7 & \sum C_{i}=17 \\ \sum C_{i}=17=17 & \mathrm{~J} 1, \mathrm{~J} 3 \rightarrow \mathrm{M} 1, & \mathrm{~J} 2, \mathrm{~J} 4 \rightarrow \mathrm{M} 2\end{array}$

$C U B_{2}$

$\mathrm{J} 1 \rightarrow \mathrm{M} 1 \quad C_{1}=1 \quad \mathrm{~J} 1 \rightarrow \mathrm{M} 1 \quad \sum C_{i}=1$

$\mathrm{J} 1 \rightarrow \mathrm{M} 2 \quad C_{1}=1$

$\mathrm{J} 2 \rightarrow \mathrm{M} 1 \quad C_{2}=6$

$\mathrm{J} 2 \rightarrow \mathrm{M} 2 \quad C_{2}=2$

$\mathrm{J} 3 \rightarrow \mathrm{M} 1 \quad C_{3}=7$

$\mathrm{J} 3 \rightarrow \mathrm{M} 2 \quad C_{3}=6 \quad \mathrm{~J} 3 \rightarrow \mathrm{M} 2 \quad \sum C_{i}=9$

$\mathrm{J} 4 \rightarrow \mathrm{M} 1 \quad C_{4}=8 \quad \mathrm{~J} 4 \rightarrow \mathrm{M} 1 \quad \sum C_{i}=17$

$\mathrm{J} 4 \rightarrow \mathrm{M} 2 \quad C_{4}=10$

$\sum C_{i}=17 \quad \mathrm{~J} 1, \mathrm{~J} 4 \rightarrow \mathrm{M} 1, \mathrm{~J} 2, \mathrm{~J} 3 \rightarrow \mathrm{M} 2$

No improvement is obtained when applying improvement heuristic $(I U B)$ to the $C U B_{1}$ schedule. Applying $I U B$ to the $C U B_{2}$ schedule reduces total flowtime; jobs 1 and 2 are interchanged and the total flowtime is 16, i.e., $\sum C_{i}=16, \mathrm{~J} 2, \mathrm{~J} 4 \rightarrow \mathrm{M} 1, \mathrm{~J} 1, \mathrm{~J} 3 \rightarrow \mathrm{M} 2$, and $U B=$ $\min \left\{C U B_{1}, C U B_{2}, I U B\right\}=16$.

The logic of the B\&B algorithm is illustrated below. For completeness, the illustration shows the computation of both lower bounds (i.e., the lower bound at each node is $\max \left\{L B_{1}\right.$, $\left.\left.L B_{2}\right\}\right)$.

1. Branch from root node where $a_{1}=0, a_{2}=0$, and earliest available machine is M1:

Node $1, \mathrm{~J} 1 \rightarrow \mathrm{M} 1, P F=1$

$L B_{1} \quad C_{2}=1.5, C_{3}=4, C_{4}=7 \quad L B_{1}=13.5$

$L B_{2} \quad C_{2}=2, C_{3}=4, C_{4}=6 \quad L B_{2}=13$ 
Node $2, \mathrm{~J} 2 \rightarrow \mathrm{M} 1, P F=2$

$L B_{1} \quad C_{1}=1, C_{3}=4, C_{4}=7$

$L B_{1}=14$

$L B_{2} \quad C_{1}=1, C_{3}=4, C_{4}=9$

$L B_{2}=16 \geq U B$, fathom

Node $3, \mathrm{~J} 3 \rightarrow \mathrm{M} 1, P F=6$

$L B_{1} \quad C_{1}=1, C_{2}=3, C_{4}=7$

$L B_{1}=17 \geq U B$, fathom

Node $4, \mathrm{~J} 4 \rightarrow \mathrm{M} 1, P F=7$

$L B_{1} \quad C_{1}=1, C_{2}=3, C_{3}=7$

$L B_{1}=18 \geq U B$, fathom

Node 5, close M1, $P F=0$

$L B_{1} \quad C_{1}=1, C_{2}=3, C_{3}=7, C_{4}=11 \quad L B_{1}=22 \geq U B$, fathom

2. Branch from Node 1 where $\mathrm{J} 1 \rightarrow \mathrm{M} 1, a_{1}=1, a_{2}=0$, and earliest available machine is $\mathrm{M} 2$ :

Node $6, \mathrm{~J} 2 \rightarrow \mathrm{M} 2, P F=3$
$L B_{1} \quad C_{3}=4, C_{4}=7$
$L B_{1}=14$
$L B_{2} \quad C_{3}=4, C_{4}=6$
$L B_{2}=13$

Node $7, \mathrm{~J} 3 \rightarrow \mathrm{M} 2, P F=4$
$L B_{1} \quad C_{2}=4, C_{4}=7$
$L B_{1}=15$
$\mathrm{LB}_{2} \quad \mathrm{C}_{2}=3, \mathrm{C}_{4}=7$
$L B_{2}=14$

Node $8, \mathrm{~J} 4 \rightarrow \mathrm{M} 2, P F=5$
$L B_{1} \quad C_{2}=5.5, C_{3}=7$
$L B_{1}=17.5 \geq U B$, fathom

Node 9, close M2, $P F=1$

$L B_{1} \quad C_{2}=6, C_{3}=9, C_{4}=13 \quad L B_{1}=29 \geq U B$, fathom

3. Branch from Node 6 where $\mathrm{J} 2 \rightarrow \mathrm{M} 2, a_{1}=1, a_{2}=2$, and earliest available machine is $\mathrm{M} 1$ :

Node $10, \mathrm{~J} 3 \rightarrow \mathrm{M} 1, P F=10$

$L B_{1} \quad C_{4}=7 \quad L B_{1}=17 \geq U B$, fathom

Node $11, \mathrm{~J} 4 \rightarrow \mathrm{M} 1, P F=11$

$L B_{1} \quad C_{3}=7 \quad L B_{1}=17 \geq U B$, fathom

Node 12, Close M1, $P F=3$

$L B_{1} \quad C_{3}=6, C_{4}=10 \quad L B_{1}=19 \geq U B$, fathom

4. Branch from Node 7 where $\mathrm{J} 3 \rightarrow \mathrm{M} 2, a_{1}=1, a_{2}=3$, and earliest available machine is $\mathrm{M} 1$ :

Node $13, \mathrm{~J} 2 \rightarrow \mathrm{M} 1, P F=10$

$L B_{1} \quad C_{4}=7 \quad L B_{1}=17 \geq U B$, fathom

Node $14, \mathrm{~J} 4 \rightarrow \mathrm{M} 1, P F=12$ 
$L B_{1} \quad C_{2}=6 \quad L B_{1}=18 \geq U B$, fathom

Node 15, Close M1, $P F=4$

$L B_{1} \quad C_{2}=6, C_{4}=10 \quad L B_{1}=20 \geq U B$, fathom

All nodes are fathomed. We have $\sum C_{i}=16, \mathrm{~J} 2, \mathrm{~J} 4 \rightarrow \mathrm{M} 1, \mathrm{~J} 1, \mathrm{~J} 3 \rightarrow \mathrm{M} 2$.

\section{References}

Adiri I., Bruno J., Frostig E. and Rinnooy Kan A.H.G., Single machine flow-time scheduling with a single breakdown, Acta Informatica, 1989, 26, 679--696.

Batun S. and Azizoglu M., Single machine scheduling with preventive maintenances, International Journal of Production Research, 2008, (forthcoming, DOI: 10.1080/00207540701636348).

Chen W.J., Minimizing total flow time in the single-machine scheduling problem with periodic maintenance, Journal of the Operational Research Society, 2006, 57, 410--415.

Chen J.S., Single-machine scheduling with flexible and periodic maintenance, Journal of the Operational Research Society, 2006, 57, 703--710.

Garey M.R. and Johnson D.S., Strong NP-Completeness results: motivation, examples and implications, Journal of the ACM, 1978, 25, 499--508.

Graves G.H. and Lee C.Y., Scheduling maintenance and semiresumable jobs on a single machine, Naval Research Logistics, 1999, 46, 845--863.

He Y., Ji M.and Cheng T.C.E., Single machine scheduling with a restricted rate-modifying activity, Naval Research Logistics, 2005, 52, 261--269.

Kacem I., Sadfi C.and El-Kamel A., Branch and bound and dynamic programming to minimize total completion times on a single machine with availability constraints, IEEE International Conference on Systems, Man and Cybernetics, 2005, 2, 1657--1662. 
Kacem I. and Chu C., Efficient branch-and-bound algorithm for minimizing the weighted sum of completion times on a single machine with one availability constraint, International Journal of Production Economics, 2008a, 112, 138--150.

Kacem I.and Chu C., Worst-case analysis of WSPT and MWSPT rules for single machine scheduling with one planned setup period, European Journal of Operational Research, 2008b, 187, 1080--1089.

Kacem I., Chu C.and Souissi A., Single-machine scheduling with an availability constraint to minimize the weighted sum of the completion times, Computers \& Operations Research, 2007, 35, 827-844.

Lee C.-Y., Machine scheduling with an availability constraint, Journal of Global Optimization, 1996, 9, 395--416.

Lee C.-Y.and Chen Z.-L., Scheduling jobs and maintenance activities on parallel machines, Naval Research Logistics, 2000, 47, 145--165.

Lee C.-Y., Lei L.and Pinedo M., Current trends in deterministic scheduling, Annals of Operations Research, 1997, 70, 1--42.

Lee C.-Y.and Leon V.J., Machine scheduling with a rate-modifying activity, European Journal of Operational Research, 2001, 128, 119-128.

Lee C.-Y.and Liman S.D., Single-machine flow-time scheduling with scheduled maintenance, Acta Informatica, 1992, 29, 375--382.

Lee C.-Y.and Liman S.D., Capacitated two-parallel machine scheduling to minimize sum of job completion times, Discrete Applied Mathematics, 1993, 41, 211--222.

Lee C.-Y. and Yu G., Parallel-machine scheduling under potential disruption, Optimization Letters, 2008, 2, 27--37. 
Mosheiov G., Minimizing the sum of job completion times on capacitated parallel machines, Mathematical and Computer Modelling, 1994, 20, 91--99.

Qi X., Chen T. and Tu F., Scheduling the maintenance on a single machine, Journal of the Operational Research Society, 1999, 50, 1071--1078.

Sadfi C., Penz B.and Rapine C., A dynamic programming algorithm for the single total completion time scheduling problem with availability constraints, 8th International Workshop on Project Management and Scheduling, 2002, Valence, Spain.

Sadfi C., Penz B., Rapine C., Blazewicz J. and Formanowicz P., An improved approximation algorithm for the single machine total completion time scheduling problem with availability constraints, European Journal of Operational Research, 2005, 161, 3--10.

Sanlaville E.and Schmidt G., Machine scheduling with availability constraints, Acta Informatica, 1998, 35, 795--811.

Schmidt G., Scheduling with limited machine availability, European Journal of Operational Research, 2000, 121, 1-15.

Wang G., Sun H.and Chu C., Preemptive scheduling with availability constraints to minimize total weighted completion times, Annals of Operations Research, 2005, 133, 183-192. 
Table 4.1 B\&B and CPLEX average CPU times (in seconds) for synchronous-periodic problem instances

\begin{tabular}{|c|c|c|c|c|c|c|c|c|c|}
\hline \multirow[b]{2}{*}{ Jobs } & \multirow{2}{*}{$\begin{array}{l}\text { Downtime } \\
\text { Frequency }\end{array}$} & \multicolumn{4}{|c|}{$p_{j} \sim \mathrm{DU}[1,30]$} & \multicolumn{4}{|c|}{$p_{j} \sim \mathrm{DU}[1,100]$} \\
\hline & & $\begin{array}{l}\text { B\&B } \\
\text { Split }\end{array}$ & $\begin{array}{l}\text { B\&B } \\
\text { Shift }\end{array}$ & $\begin{array}{c}\text { CPLEX } \\
I_{1}\end{array}$ & $\begin{array}{c}\text { CPLEX } \\
I_{P_{2}}\end{array}$ & $\begin{array}{l}\text { B\&B } \\
\text { Split }\end{array}$ & $\begin{array}{l}\text { B\&B } \\
\text { Shift }\end{array}$ & $\begin{array}{c}\text { CPLEX } \\
I_{P_{1}}\end{array}$ & $\begin{array}{c}\text { CPLEX } \\
I_{2}\end{array}$ \\
\hline \multirow{3}{*}{10} & 1 & 0,0 & 0,0 & 0,1 & 0,1 & 0,0 & 0,0 & 0,1 & 0,1 \\
\hline & 3 & 0,0 & 0,0 & 0,3 & 0,2 & 0,0 & 0,0 & 0,3 & 0,2 \\
\hline & 5 & 0,0 & $\overline{0,0}$ & 0,4 & 0,4 & 0,0 & 0,0 & 0,4 & 0,3 \\
\hline \multirow{3}{*}{15} & 1 & 0,1 & 0,0 & 1,8 & 1,2 & 0,1 & 0,0 & 2,5 & 1,3 \\
\hline & 3 & 0,1 & $\overline{0,1}$ & 16,3 & 4,1 & 0,1 & 0,1 & 18,1 & 4,2 \\
\hline & 5 & 0,1 & 0,1 & 19,0 & 7,6 & 0,1 & 0,1 & 27,1 & 7,5 \\
\hline \multirow{3}{*}{20} & 1 & 1,1 & 0,3 & 90,6 & 33,2 & 1,3 & 0,3 & 64,0 & 33,0 \\
\hline & 3 & 1,1 & 1,0 & 734,6 & 132,2 & 1,3 & $\overline{1,1}$ & 710,9 & 115,5 \\
\hline & 5 & 0,9 & 1,1 & $2.000,1$ & 220,1 & 1,1 & 1,4 & $2.464,6$ & 243,9 \\
\hline 25 & 1 & 27,2 & 3,7 & $*$ & 582,4 & 38,8 & 6,5 & * & 718,4 \\
\hline
\end{tabular}

Table 4.2 B\&B and CPLEX average CPU times (in seconds) for asynchronous problem instances

\begin{tabular}{|c|c|c|c|c|c|c|c|c|c|}
\hline \multirow[b]{2}{*}{ Jobs } & \multirow[b]{2}{*}{$\begin{array}{l}\text { Downtime } \\
\text { Frequency }\end{array}$} & \multicolumn{4}{|c|}{$p_{j} \sim \operatorname{DU}[1,30]$} & \multicolumn{4}{|c|}{$p_{j} \sim \mathrm{DU}[1,100]$} \\
\hline & & $\begin{array}{l}\text { B\&B } \\
\text { Split }\end{array}$ & $\begin{array}{l}\text { B\&B } \\
\text { Shift }\end{array}$ & $\begin{array}{c}\text { CPLEX } \\
I_{1}\end{array}$ & $\begin{array}{c}\text { CPLEX } \\
I P_{2}\end{array}$ & $\begin{array}{l}\text { B\&B } \\
\text { Split }\end{array}$ & $\begin{array}{l}\text { B\&B } \\
\text { Shift }\end{array}$ & $\begin{array}{c}\text { CPLEX } \\
I P_{1}\end{array}$ & $\begin{array}{c}\text { CPLEX } \\
\boldsymbol{I P}_{2}\end{array}$ \\
\hline \multirow{3}{*}{10} & 1 & 0,0 & 0,0 & 0,1 & 0,1 & 0,0 & 0,0 & 0,1 & 0,1 \\
\hline & 3 & 0,0 & 0,0 & 0,3 & 0,2 & 0,0 & 0,0 & 0,3 & 0,2 \\
\hline & 5 & 0,0 & 0,0 & 0,7 & 0,4 & 0,0 & 0,0 & 0,8 & $\overline{0,4}$ \\
\hline \multirow{3}{*}{15} & 1 & 0,1 & 0,1 & 1,9 & 1,1 & 0,1 & 0,1 & 2,0 & 1,0 \\
\hline & 3 & 0,1 & 0,1 & 7,8 & 3,1 & 0,1 & 0,1 & 9,2 & 3,2 \\
\hline & 5 & 0,1 & 0,1 & 21,3 & 5,2 & 0,1 & 0,1 & 20,6 & $\overline{5,1}$ \\
\hline \multirow{3}{*}{20} & 1 & 2,6 & 1,8 & 97,9 & 31,5 & 2,8 & 1,7 & 123,4 & 28,9 \\
\hline & 3 & 1,9 & 2,1 & 632,5 & 87,6 & 1,7 & 2,0 & 545,9 & 73,3 \\
\hline & 5 & 2,3 & 3,2 & $1.287,9$ & 166,8 & 2,2 & 2,9 & $1.836,4$ & 152,7 \\
\hline 25 & 1 & 70,3 & 34,6 & * & 554,0 & 72,1 & 34,9 & * & 747,5 \\
\hline
\end{tabular}




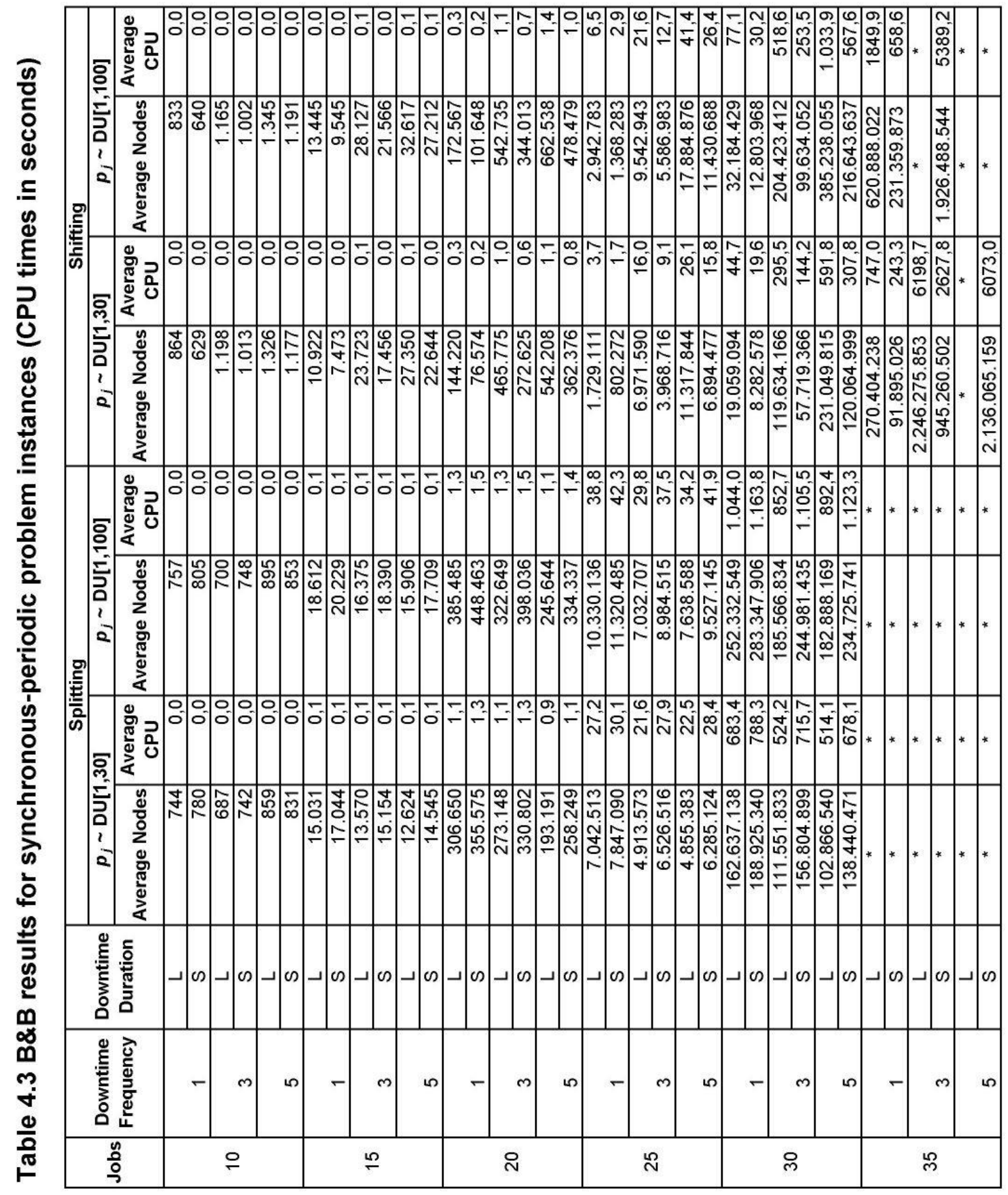




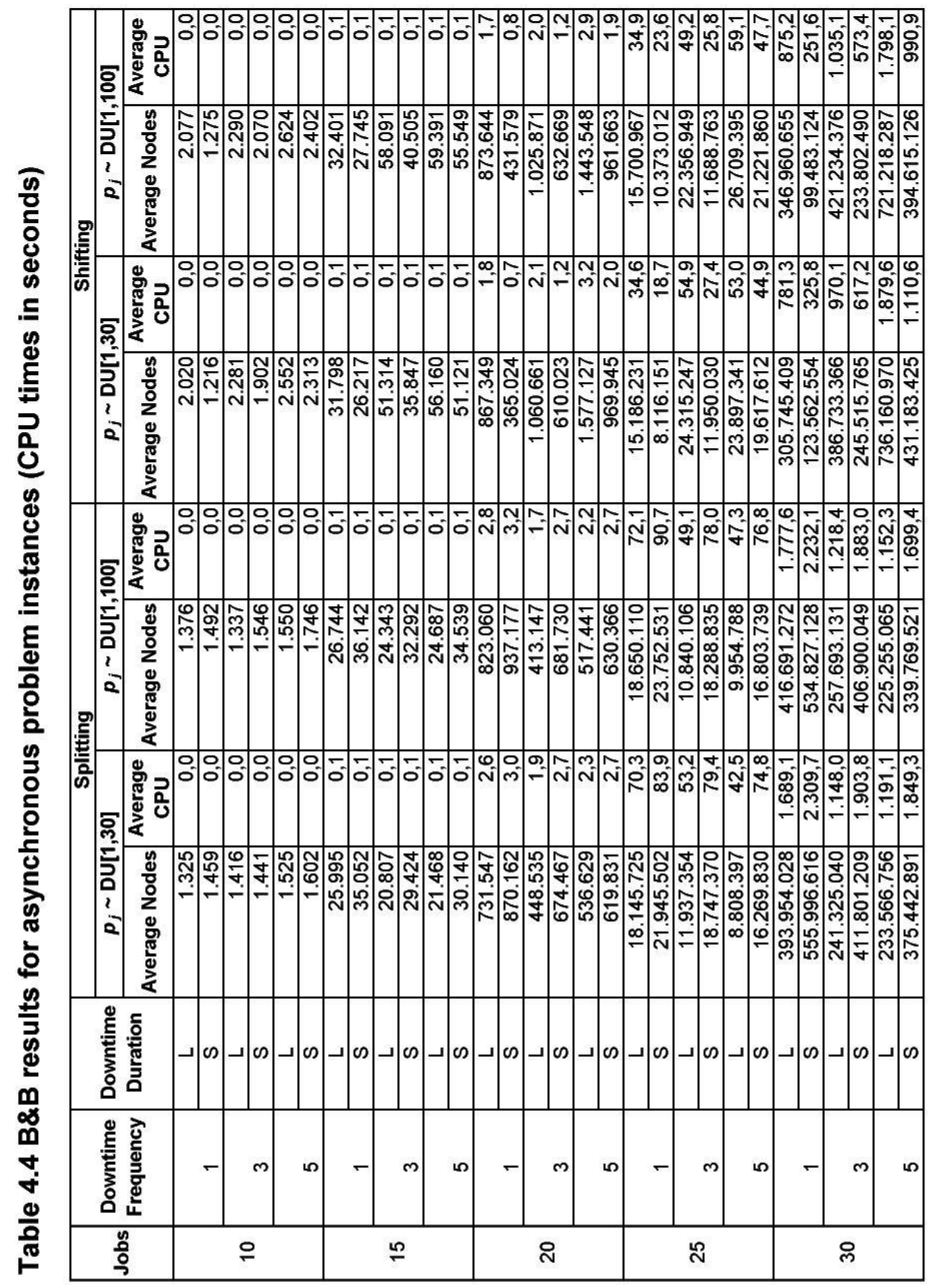


Table 4.5 Upper bound results for synchronous-periodic problem instances

\begin{tabular}{|c|c|c|c|c|c|c|c|c|c|c|c|c|c|c|}
\hline \multirow{3}{*}{ Jobs } & \multirow{3}{*}{$\begin{array}{l}\text { Downtime } \\
\text { Frequency }\end{array}$} & \multirow{3}{*}{$\begin{array}{c}\text { Downtime } \\
\text { Duration }\end{array}$} & \multicolumn{6}{|c|}{$p_{j} \sim \operatorname{DU}[1,30]$} & \multicolumn{6}{|c|}{$p_{j} \sim \mathrm{DU}[1,100]$} \\
\hline & & & \multicolumn{2}{|c|}{$\mathrm{CUB}_{1}$} & \multicolumn{2}{|c|}{$\mathrm{CUB}_{2}$} & \multicolumn{2}{|c|}{ IUB } & \multicolumn{2}{|c|}{$\mathrm{CUB}_{1}$} & \multicolumn{2}{|c|}{$\mathrm{CUB}_{2}$} & \multicolumn{2}{|c|}{ IUB } \\
\hline & & & Avg & Max & Avg & Max & Avg & Max & Avg & $\operatorname{Max}$ & Avg & $\operatorname{Max}$ & Avg & Max \\
\hline \multirow{6}{*}{10} & \multirow[b]{2}{*}{1} & $L$ & $3,3 \%$ & $6,9 \%$ & $3,3 \%$ & $6,9 \%$ & $0,3 \%$ & $1,1 \%$ & $4,4 \%$ & $7,0 \%$ & $4,4 \%$ & $7,0 \%$ & $0,5 \%$ & $4,4 \%$ \\
\hline & & $\mathrm{S}$ & $1,7 \%$ & $3,6 \%$ & $1,7 \%$ & $3,6 \%$ & $0,3 \%$ & $1,2 \%$ & $2,3 \%$ & $3,7 \%$ & $2,3 \%$ & $3,7 \%$ & $0,5 \%$ & $2,3 \%$ \\
\hline & \multirow[b]{2}{*}{3} & $\mathrm{~L}$ & $3,8 \%$ & $10,8 \%$ & $3,8 \%$ & $10,8 \%$ & $0,5 \%$ & $2,7 \%$ & $4,2 \%$ & $10,9 \%$ & $4,2 \%$ & $10,9 \%$ & $0,1 \%$ & $0,5 \%$ \\
\hline & & $\mathrm{S}$ & $2,1 \%$ & $6,2 \%$ & $2,1 \%$ & $6,2 \%$ & $0,3 \%$ & $1,3 \%$ & $2,3 \%$ & $6,4 \%$ & $2,3 \%$ & $6,4 \%$ & $0,1 \%$ & $0,6 \%$ \\
\hline & \multirow[b]{2}{*}{5} & $\mathrm{~L}$ & $3,5 \%$ & $5,0 \%$ & $3,5 \%$ & $5,0 \%$ & $0,3 \%$ & $2,2 \%$ & $3,8 \%$ & $7,7 \%$ & $3,8 \%$ & $7,7 \%$ & $0,2 \%$ & $1,2 \%$ \\
\hline & & $\mathrm{S}$ & $2,1 \%$ & $3,0 \%$ & $2,1 \%$ & $3,0 \%$ & $0,2 \%$ & $1,2 \%$ & $2,4 \%$ & $4,9 \%$ & $2,4 \%$ & $4,9 \%$ & $0,2 \%$ & $1,2 \%$ \\
\hline \multirow{6}{*}{15} & \multirow[b]{2}{*}{1} & $\mathrm{~L}$ & $2,2 \%$ & $3,6 \%$ & $2,2 \%$ & $3,6 \%$ & $0,4 \%$ & $1,1 \%$ & $2,3 \%$ & $3,9 \%$ & $2,3 \%$ & $3,9 \%$ & $0,6 \%$ & $2,1 \%$ \\
\hline & & $\mathrm{S}$ & $1,0 \%$ & $1,7 \%$ & $1,0 \%$ & $1,7 \%$ & $0,3 \%$ & $0,9 \%$ & $1,1 \%$ & $1,9 \%$ & $1,1 \%$ & $1,9 \%$ & $0,4 \%$ & $1,1 \%$ \\
\hline & \multirow[b]{2}{*}{3} & $\mathrm{~L}$ & $2,2 \%$ & $4,8 \%$ & $2,2 \%$ & $4,8 \%$ & $0,3 \%$ & $1,6 \%$ & $2,3 \%$ & $4,7 \%$ & $2,3 \%$ & $4,7 \%$ & $0,5 \%$ & $2,2 \%$ \\
\hline & & $\mathrm{S}$ & $1,2 \%$ & $2,6 \%$ & $1,2 \%$ & $2,6 \%$ & $0,2 \%$ & $0,9 \%$ & $1,3 \%$ & $2,7 \%$ & $1,3 \%$ & $2,7 \%$ & $0,4 \%$ & $1,6 \%$ \\
\hline & \multirow[b]{2}{*}{5} & $\mathrm{~L}$ & $2,6 \%$ & $5,7 \%$ & $2,6 \%$ & $5,7 \%$ & $0,3 \%$ & $1,2 \%$ & $3,1 \%$ & $6,2 \%$ & $3,1 \%$ & $6,2 \%$ & $1,1 \%$ & $3,8 \%$ \\
\hline & & $\mathrm{S}$ & $1,5 \%$ & $3,3 \%$ & $1,5 \%$ & $3,3 \%$ & $0,1 \%$ & $0,7 \%$ & $1,9 \%$ & $3,8 \%$ & $1,9 \%$ & $3,8 \%$ & $0,6 \%$ & $2,5 \%$ \\
\hline \multirow{6}{*}{20} & \multirow[b]{2}{*}{1} & $\mathrm{~L}$ & $1,4 \%$ & $2,3 \%$ & $1,4 \%$ & $2,3 \%$ & $0,1 \%$ & $0,7 \%$ & $1,5 \%$ & $2,4 \%$ & $1,5 \%$ & $2,4 \%$ & $0,2 \%$ & $0,8 \%$ \\
\hline & & $\mathrm{S}$ & $0,7 \%$ & $1,1 \%$ & $0,7 \%$ & $1,1 \%$ & $0,0 \%$ & $0,2 \%$ & $0,8 \%$ & $1,2 \%$ & $0,8 \%$ & $1,2 \%$ & $0,2 \%$ & $0,6 \%$ \\
\hline & \multirow[b]{2}{*}{3} & $\mathrm{~L}$ & $1,4 \%$ & $2,0 \%$ & $1,4 \%$ & $2,0 \%$ & $0,2 \%$ & $1,0 \%$ & $1,7 \%$ & $3,0 \%$ & $1,7 \%$ & $3,0 \%$ & $0,3 \%$ & $1,1 \%$ \\
\hline & & $\mathrm{S}$ & $0,7 \%$ & $1,0 \%$ & $0,7 \%$ & $1,0 \%$ & $0,1 \%$ & $0,4 \%$ & $0,9 \%$ & $1,7 \%$ & $0,9 \%$ & $1,7 \%$ & $0,2 \%$ & $0,7 \%$ \\
\hline & \multirow[b]{2}{*}{5} & $\mathrm{~L}$ & $1,9 \%$ & $3,1 \%$ & $1,9 \%$ & $3,1 \%$ & $0,1 \%$ & $0,9 \%$ & $2,1 \%$ & $3,6 \%$ & $2,1 \%$ & $3,6 \%$ & $0,1 \%$ & $0,8 \%$ \\
\hline & & $\mathrm{S}$ & $1,0 \%$ & $1,7 \%$ & $1,0 \%$ & $1,7 \%$ & $0,1 \%$ & $0,6 \%$ & $1,2 \%$ & $2,1 \%$ & $1,2 \%$ & $2,1 \%$ & $0,2 \%$ & $0,5 \%$ \\
\hline \multirow{6}{*}{25} & \multirow[b]{2}{*}{1} & $\mathrm{~L}$ & $1,0 \%$ & $1,5 \%$ & $1,0 \%$ & $1,5 \%$ & $0,2 \%$ & $0,4 \%$ & $0,9 \%$ & $1,6 \%$ & $0,9 \%$ & $1,6 \%$ & $0,2 \%$ & $0,2 \%$ \\
\hline & & $S$ & $0,5 \%$ & $0,8 \%$ & $0,5 \%$ & $0,8 \%$ & $0,1 \%$ & $0,3 \%$ & $0,5 \%$ & $0,8 \%$ & $0,5 \%$ & $0,8 \%$ & $0,2 \%$ & $0,2 \%$ \\
\hline & & $\mathrm{L}$ & $1,4 \%$ & $2,0 \%$ & $1,4 \%$ & $2,0 \%$ & $0,2 \%$ & $0,7 \%$ & $1,4 \%$ & $2,0 \%$ & $1,4 \%$ & $2,0 \%$ & $0,4 \%$ & $0,8 \%$ \\
\hline & 3 & S & $0,7 \%$ & $1,1 \%$ & $0,7 \%$ & $1,1 \%$ & $0,2 \%$ & $0,4 \%$ & $0,7 \%$ & $1,1 \%$ & $0,7 \%$ & $1,1 \%$ & $0,2 \%$ & $0,5 \%$ \\
\hline & & $\mathrm{L}$ & $1,2 \%$ & $2,6 \%$ & $1,2 \%$ & $2,6 \%$ & $0,1 \%$ & $0,3 \%$ & $1,0 \%$ & $2,0 \%$ & $1,0 \%$ & $2,0 \%$ & $0,3 \%$ & $0,7 \%$ \\
\hline & 5 & $\mathrm{~S}$ & $0,6 \%$ & $1,5 \%$ & $0,6 \%$ & $1,5 \%$ & $0,1 \%$ & $0,4 \%$ & $0,5 \%$ & $1,1 \%$ & $0,5 \%$ & $1,1 \%$ & $0,1 \%$ & $0,4 \%$ \\
\hline & & $\mathrm{L}$ & $0,7 \%$ & $1,2 \%$ & $0,7 \%$ & $1,2 \%$ & $0,1 \%$ & $0,3 \%$ & $0,7 \%$ & $1,2 \%$ & $0,7 \%$ & $1,2 \%$ & $0,1 \%$ & $0,2 \%$ \\
\hline & 1 & $\mathrm{~S}$ & $0,3 \%$ & $0,6 \%$ & $0,3 \%$ & $0,6 \%$ & $0,1 \%$ & $0,1 \%$ & $0,3 \%$ & $0,6 \%$ & $0,3 \%$ & $0,6 \%$ & $0,1 \%$ & $0,2 \%$ \\
\hline 3 & & $\mathrm{~L}$ & $0,9 \%$ & $1,3 \%$ & $0,9 \%$ & $1,3 \%$ & $0,1 \%$ & $0,5 \%$ & $1,0 \%$ & $1,6 \%$ & $1,0 \%$ & $1,6 \%$ & $0,1 \%$ & $0,5 \%$ \\
\hline 30 & 3 & $\mathrm{~S}$ & $0,5 \%$ & $0,6 \%$ & $0,5 \%$ & $0,6 \%$ & $0,1 \%$ & $0,3 \%$ & $0,5 \%$ & $0,9 \%$ & $0,5 \%$ & $0,9 \%$ & $0,1 \%$ & $0,2 \%$ \\
\hline & & $\mathrm{L}$ & $0,9 \%$ & $1,3 \%$ & $0,9 \%$ & $1,3 \%$ & $0,1 \%$ & $0,3 \%$ & $0,9 \%$ & $1,7 \%$ & $0,9 \%$ & $1,7 \%$ & $0,1 \%$ & $0,5 \%$ \\
\hline & 5 & $\mathrm{~S}$ & $0,5 \%$ & $0,7 \%$ & $0,5 \%$ & $0,7 \%$ & $0,0 \%$ & $0,2 \%$ & $0,5 \%$ & $0,9 \%$ & $0,5 \%$ & $0,9 \%$ & $0,1 \%$ & $0,3 \%$ \\
\hline & & Overall & $1,4 \%$ & $10,8 \%$ & $1,4 \%$ & $10,8 \%$ & $0,2 \%$ & $2,7 \%$ & $1,6 \%$ & $10,9 \%$ & $1,6 \%$ & $10,9 \%$ & $0,3 \%$ & $4,4 \%$ \\
\hline
\end{tabular}


Table 4.6 Upper bound results for asynchronous problem instances

\begin{tabular}{|c|c|c|c|c|c|c|c|c|c|c|c|c|c|c|}
\hline \multirow{3}{*}{ Jobs } & \multirow{3}{*}{$\begin{array}{l}\text { Downtime } \\
\text { Frequency }\end{array}$} & \multirow{3}{*}{$\begin{array}{c}\text { Downtime } \\
\text { Duration }\end{array}$} & \multicolumn{6}{|c|}{$p_{j} \sim \operatorname{DU}[1,30]$} & \multicolumn{6}{|c|}{$p_{j} \sim \operatorname{DU}[1,100]$} \\
\hline & & & \multicolumn{2}{|c|}{$\mathrm{CUB}_{1}$} & \multicolumn{2}{|c|}{$\mathrm{CUB}_{2}$} & \multicolumn{2}{|c|}{ IUB } & \multicolumn{2}{|c|}{$\mathrm{CUB}_{1}$} & \multicolumn{2}{|c|}{$\mathrm{CUB}_{2}$} & \multicolumn{2}{|c|}{ IUB } \\
\hline & & & Avg & Max & Avg & Max & Avg & Max & Avg & Max & Avg & Max & Avg & Max \\
\hline \multirow{6}{*}{10} & \multirow[b]{2}{*}{1} & $\mathrm{~L}$ & $2,8 \%$ & $7,3 \%$ & $2,9 \%$ & $7,3 \%$ & $0,3 \%$ & $2,4 \%$ & $3,6 \%$ & $7,0 \%$ & $2,9 \%$ & $6,5 \%$ & $0,3 \%$ & $1,1 \%$ \\
\hline & & $\mathrm{S}$ & $1,7 \%$ & $3,9 \%$ & $1,3 \%$ & $3,5 \%$ & $0,2 \%$ & $1,5 \%$ & $1,7 \%$ & $4,1 \%$ & $1,3 \%$ & $3,0 \%$ & $0,2 \%$ & $1,6 \%$ \\
\hline & \multirow[b]{2}{*}{3} & L & $6,0 \%$ & $18,5 \%$ & $5,0 \%$ & $12,0 \%$ & $0,7 \%$ & $3,9 \%$ & $6,2 \%$ & $12,5 \%$ & $5,0 \%$ & $11,2 \%$ & $0,8 \%$ & $2,6 \%$ \\
\hline & & $S$ & $2,3 \%$ & $5,0 \%$ & $2,3 \%$ & $5,0 \%$ & $0,3 \%$ & $1,0 \%$ & $2,5 \%$ & $4,8 \%$ & $2,0 \%$ & $4,3 \%$ & $0,7 \%$ & $1,9 \%$ \\
\hline & & $\mathrm{L}$ & $4,1 \%$ & $13,8 \%$ & $4,1 \%$ & $10,2 \%$ & $0,9 \%$ & $2,2 \%$ & $4,0 \%$ & $13,7 \%$ & $4,1 \%$ & $11,6 \%$ & $1,1 \%$ & $6,4 \%$ \\
\hline & & $\mathrm{S}$ & $3,4 \%$ & $7,4 \%$ & $3,3 \%$ & $8,2 \%$ & $0,6 \%$ & $2,4 \%$ & $2,6 \%$ & $5,9 \%$ & $2,7 \%$ & $5,7 \%$ & $0,6 \%$ & $2,4 \%$ \\
\hline \multirow{6}{*}{15} & & $\mathrm{~L}$ & $1,1 \%$ & $3,4 \%$ & $1,2 \%$ & $3,4 \%$ & $0,1 \%$ & $0,3 \%$ & $1,7 \%$ & $3,4 \%$ & $2,0 \%$ & $4,6 \%$ & $0,4 \%$ & $1,3 \%$ \\
\hline & & $\mathrm{S}$ & $1,2 \%$ & $2,4 \%$ & $1,2 \%$ & $2,4 \%$ & $0,3 \%$ & $0,7 \%$ & $1,2 \%$ & $2,6 \%$ & $1,2 \%$ & $2,6 \%$ & $0,3 \%$ & $0,8 \%$ \\
\hline & & $\mathrm{L}$ & $2,3 \%$ & $7,6 \%$ & $1,8 \%$ & $7,7 \%$ & $0,1 \%$ & $0,7 \%$ & $2,0 \%$ & $4,0 \%$ & $1,9 \%$ & $3,6 \%$ & $0,5 \%$ & $1,8 \%$ \\
\hline & & S & $1,4 \%$ & $4,7 \%$ & $1,3 \%$ & $3,1 \%$ & $0,3 \%$ & $1,3 \%$ & $1,5 \%$ & $5,1 \%$ & $1,5 \%$ & $3,6 \%$ & $0,4 \%$ & $1,3 \%$ \\
\hline & & $\mathrm{L}$ & $2,4 \%$ & $5,1 \%$ & $2,2 \%$ & $4,5 \%$ & $0,8 \%$ & $1,7 \%$ & $3,0 \%$ & $5,7 \%$ & $2,5 \%$ & $5 \%$ & $0,8 \%$ & $2,0 \%$ \\
\hline & & S & $2,2 \%$ & $4,0 \%$ & $2,1 \%$ & $4,0 \%$ & $0,4 \%$ & $1,5 \%$ & $2,5 \%$ & $4,7 \%$ & $2,2 \%$ & $4 \%$ & $0,3 \%$ & $0,8 \%$ \\
\hline \multirow{6}{*}{20} & & L & $0,7 \%$ & $1,6 \%$ & $0,3 \%$ & $1,6 \%$ & $0,1 \%$ & $0,5 \%$ & $0,6 \%$ & $1,7 \%$ & $0,6 \%$ & $5 \%$ & $0,1 \%$ & $0,5 \%$ \\
\hline & & S & $0,4 \%$ & $0,9 \%$ & $0,4 \%$ & $0,9 \%$ & $0,1 \%$ & $0,2 \%$ & $0,5 \%$ & $1,0 \%$ & $0,5 \%$ & $0 \%$ & $0,1 \%$ & $0,3 \%$ \\
\hline & \multirow[b]{2}{*}{3} & $\mathrm{~L}$ & $1,9 \%$ & $4,4 \%$ & $1,4 \%$ & $2,8 \%$ & $0,3 \%$ & $0,8 \%$ & $2,3 \%$ & $3,8 \%$ & $1,9 \%$ & $6 \%$ & $0,4 \%$ & $1,1 \%$ \\
\hline & & S & $1,1 \%$ & $3,0 \%$ & $1,0 \%$ & $2,9 \%$ & $0,3 \%$ & $0,7 \%$ & $1,2 \%$ & $3,1 \%$ & $1,2 \%$ & $2,9 \%$ & $0,2 \%$ & $0,4 \%$ \\
\hline & \multirow[b]{2}{*}{5} & L & $1,6 \%$ & $3,6 \%$ & $1,4 \%$ & $2,2 \%$ & $0,3 \%$ & $0,6 \%$ & $2,2 \%$ & $6,0 \%$ & $2,0 \%$ & $4,8 \%$ & $0,7 \%$ & $3,2 \%$ \\
\hline & & S & $1,2 \%$ & $1,9 \%$ & $0,9 \%$ & $1,9 \%$ & $0,3 \%$ & $0,6 \%$ & $1,2 \%$ & $2,0 \%$ & $1,0 \%$ & $1,8 \%$ & $0,1 \%$ & $0,4 \%$ \\
\hline \multirow{6}{*}{25} & & $\mathrm{~L}$ & $0,9 \%$ & $2,0 \%$ & $0,8 \%$ & $1,3 \%$ & $0,0 \%$ & $0,2 \%$ & $1,0 \%$ & $1,9 \%$ & $0,8 \%$ & $1,3 \%$ & $0,1 \%$ & $0,3 \%$ \\
\hline & & S & $0,4 \%$ & $1,3 \%$ & $0,4 \%$ & $1,1 \%$ & $0,2 \%$ & $0,6 \%$ & $0,5 \%$ & $1,3 \%$ & $0,4 \%$ & $1,2 \%$ & $0,1 \%$ & $0,3 \%$ \\
\hline & & L & $1,2 \%$ & $2,1 \%$ & $1,0 \%$ & $1,6 \%$ & $0,2 \%$ & $0,5 \%$ & $1,4 \%$ & $2,8 \%$ & $1,3 \%$ & $2,8 \%$ & $0,3 \%$ & $0,9 \%$ \\
\hline & 3 & S & $0,6 \%$ & $1,0 \%$ & $0,6 \%$ & $1,7 \%$ & $0,1 \%$ & $0,6 \%$ & $0,6 \%$ & $1,0 \%$ & $0,6 \%$ & $1,5 \%$ & $0,2 \%$ & $0,4 \%$ \\
\hline & & $\mathrm{L}$ & $1,4 \%$ & $2,5 \%$ & $1,2 \%$ & $2,5 \%$ & $0,4 \%$ & $1,6 \%$ & $1,3 \%$ & $3,1 \%$ & $1,4 \%$ & $3,1 \%$ & $0,4 \%$ & $1,1 \%$ \\
\hline & 5 & $\mathrm{~S}$ & $0,9 \%$ & $1,8 \%$ & $0,8 \%$ & $1,5 \%$ & $0,1 \%$ & $0,2 \%$ & $0,9 \%$ & $2,2 \%$ & $0,7 \%$ & $1,1 \%$ & $0,2 \%$ & $0,3 \%$ \\
\hline & & $\mathrm{L}$ & $0,6 \%$ & $1,2 \%$ & $0,6 \%$ & $1,2 \%$ & $0,1 \%$ & $0,3 \%$ & $0,6 \%$ & $1,3 \%$ & $0,5 \%$ & $0,9 \%$ & $0,1 \%$ & $0,3 \%$ \\
\hline & 1 & S & $0,2 \%$ & $0,5 \%$ & $0,2 \%$ & $0,5 \%$ & $0,0 \%$ & $0,1 \%$ & $0,3 \%$ & $0,5 \%$ & $0,2 \%$ & $0,5 \%$ & $0,0 \%$ & $0,2 \%$ \\
\hline & & $\mathrm{L}$ & $1,1 \%$ & $2,1 \%$ & $0,9 \%$ & $2,1 \%$ & $0,2 \%$ & $0,6 \%$ & $1,0 \%$ & $1,6 \%$ & $0,9 \%$ & $1,6 \%$ & $0,2 \%$ & $0,9 \%$ \\
\hline 30 & 3 & S & $0,4 \%$ & $1,0 \%$ & $0,4 \%$ & $1,0 \%$ & $0,1 \%$ & $0,5 \%$ & $0,5 \%$ & $1,0 \%$ & $0,5 \%$ & $1,0 \%$ & $0,1 \%$ & $0,2 \%$ \\
\hline & & L & $0,9 \%$ & $2,1 \%$ & $0,6 \%$ & $1,5 \%$ & $0,2 \%$ & $0,4 \%$ & $1,0 \%$ & $2,3 \%$ & $0,8 \%$ & $1,9 \%$ & $0,1 \%$ & $0,7 \%$ \\
\hline & 5 & $\mathrm{~S}$ & $0,5 \%$ & $1,1 \%$ & $0.4 \%$ & $0.9 \%$ & $0.1 \%$ & $0,4 \%$ & $0.6 \%$ & $1.1 \%$ & $0.6 \%$ & $1,3 \%$ & $0,2 \%$ & $0.5 \%$ \\
\hline & & Overall & $16 \%$ & $18.5 \%$ & $14 \%$ & $12.0 \%$ & $0,3 \%$ & $3.9 \%$ & $1.7 \%$ & $13.7 \%$ & $15 \%$ & $116 \%$ & $0.3 \%$ & $6.4 \%$ \\
\hline
\end{tabular}


Figure 1.1 Two example schedules with planned downtimes (shaded sections)

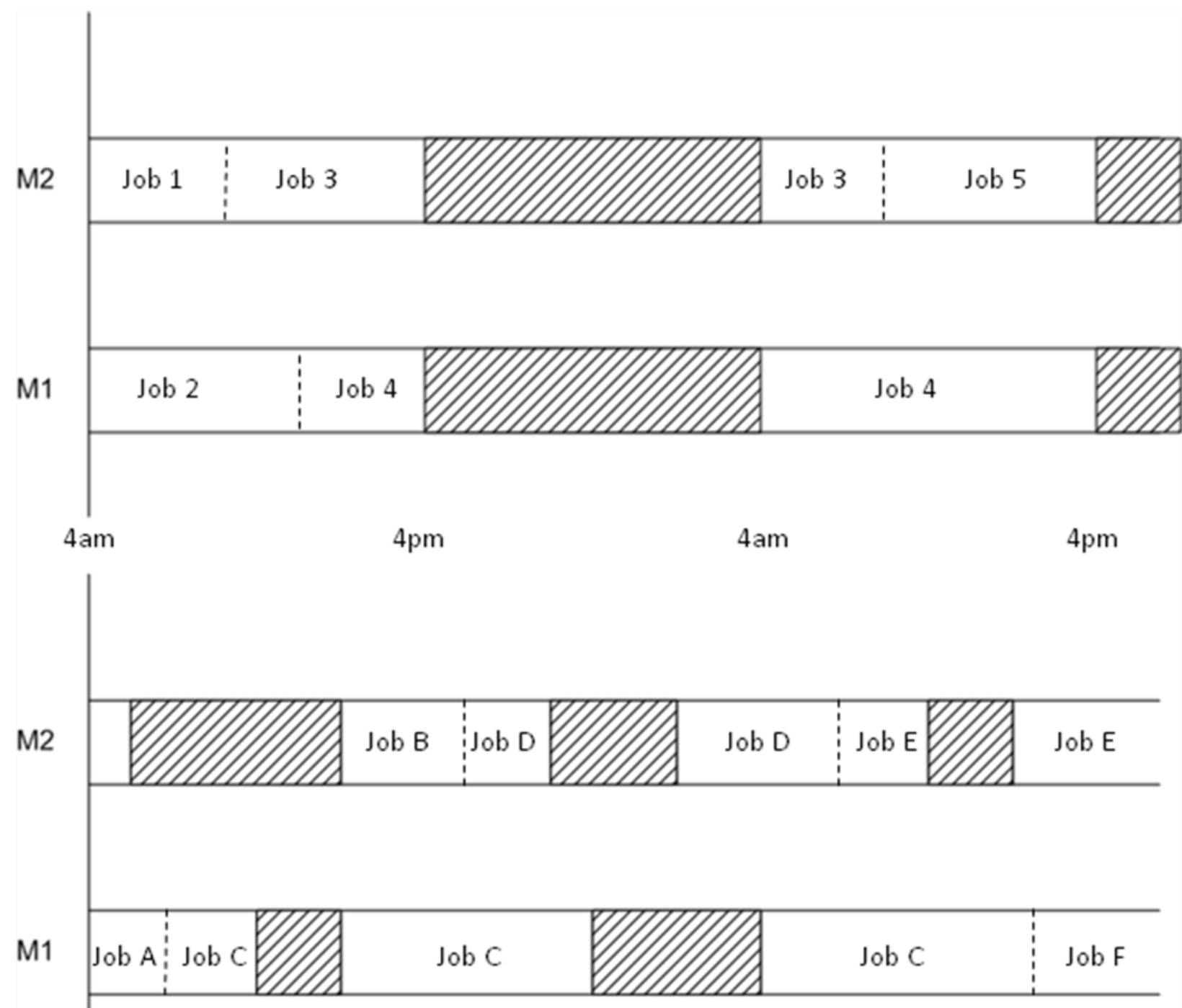

